

\title{
L'écart d'âge entre conjoints
}

Jean-François Mignot

\section{To cite this version:}

Jean-François Mignot. L'écart d'âge entre conjoints. Revue française de sociologie, 2010, 51 (2), pp.281-320. 10.3917/rfs.512.0281 . halshs-01326705

\section{HAL Id: halshs-01326705 \\ https://shs.hal.science/halshs-01326705}

Submitted on 5 Jun 2016

HAL is a multi-disciplinary open access archive for the deposit and dissemination of scientific research documents, whether they are published or not. The documents may come from teaching and research institutions in France or abroad, or from public or private research centers.
L'archive ouverte pluridisciplinaire $\mathbf{H A L}$, est destinée au dépôt et à la diffusion de documents scientifiques de niveau recherche, publiés ou non, émanant des établissements d'enseignement et de recherche français ou étrangers, des laboratoires publics ou privés. 


\title{
Jean-François MIGNOT
}

\section{L'écart d'âge entre conjoints*}

\begin{abstract}
RÉSUMÉ
Pourquoi, dans la quasi-totalité des sociétés humaines connues, les hommes sont-ils en moyenne plus âgés que leur conjointe ? Et comment - et pourquoi - l'écart d'âge moyen entre conjoints varie-t-il avec le développement socioéconomique (au fil du temps aussi bien qu'entre sociétés) et, entre couples, avec les âges des hommes et des femmes à la mise en couple, ainsi qu'avec la position sociale des hommes ? Pour le savoir, nous proposons une revue de la littérature empirique internationale sur le sujet, ainsi qu'un modèle explicatif indiquant quelles seraient les «bonnes raisons» que pourraient avoir les individus de former des couples comportant un écart d'âge entre conjoints au profit de l'homme. Un test empirique de ce modèle explicatif portant sur les couples formés en France entre 1978 et 1998 suggère que ce dernier possède un bon pouvoir explicatif, même si, en lui-même, il ne permet pas d'expliquer l'inversion du gradient social de l'écart d'âge entre conjoints survenue dans la seconde moitié du $\mathrm{XX}^{\mathrm{e}}$ siècle, qui est sans doute due à la prolongation de la scolarité des hommes.
\end{abstract}

Au sein des couples, l'homme est généralement plus âgé que la femme. Il semble même que ni les historiens, ni les anthropologues, ni les sociologues ou démographes n'aient rencontré de société humaine échappant durablement à cette régularité : presque partout, presque toujours, les couples connaissent en moyenne un écart d'âge entre conjoints au profit de l'homme. Le caractère apparemment universel de cet écart pose au chercheur en sciences sociales une question simple : pourquoi existe-t-il au sein des couples un écart d'âge moyen entre conjoints au profit de l'homme, plutôt qu'aucun écart d'âge, ou plutôt qu'un écart d'âge moyen au profit de la femme?

L'écart d'âge entre conjoints peut effectivement apparaître énigmatique à deux égards au moins. D'une part, comme « chaque âge a ses plaisirs » et ses centres d'intérêt spécifiques, et qu'au sein de leurs couples les femmes (ainsi que, dans une certaine mesure, les hommes) accordent une grande importance à la qualité des communications verbales entre conjoints (Davis, 1998), on pourrait imaginer que les individus cherchent à se mettre en couple avec un conjoint d'un âge le plus proche possible du leur, ce qui conduirait (en moyenne) les conjoints à avoir entre eux un écart d'âge nul. D'autre part, on pourrait imaginer que les individus cherchent à se mettre en couple avec un conjoint d'un âge tel qu'il leur permette de minimiser leur risque de veuvage ;

* Je tiens à remercier les membres du jury de ma thèse de doctorat, dont cet article est tiré, ainsi que les lecteurs du comité de la Revue française de sociologie pour leurs conseils. 
étant donné que, dans la plupart des sociétés humaines (notamment les sociétés occidentales contemporaines), les hommes meurent plus précocement que les femmes, les conjoints seraient alors conduits à préférer un écart d'âge entre conjoints au profit de la femme (Choo et Siow, 2005). Mais ce n'est pas ce que l'on observe. Alors, pourquoi les femmes et les hommes semblent-ils universellement préférer se mettre en couple avec un conjoint - respectivement - plus âgé et plus jeune qu'eux ?

Pour répondre à cette question, nous présentons un bilan de la littérature empirique internationale sur le sujet, puis nous proposons un modèle susceptible d'expliquer non seulement l'existence de l'écart d'âge entre conjoints, mais aussi les variations de son ampleur entre sociétés et entre couples d'une même société. Nous procédons ensuite à des analyses empiriques destinées à évaluer dans quelle mesure ce modèle dispose d'un pouvoir explicatif en ce qui concerne les couples formés dans la France contemporaine.

\section{L'écart d'âge entre conjoints au profit de l'homme : un phénomène universel, mais d'intensité variable}

L'écart d'âge entre conjoints au profit de l'homme est la différence entre l'âge de l'homme et l'âge de la femme d'un couple (marié ou non). Dans les sociétés humaines, l'écart d'âge moyen entre conjoints au profit de l'homme apparaît presque toujours positif. Par exemple, dans des sociétés du passé, l'écart d'âge moyen entre époux au profit de l'homme est de trois à quatre ans dans deux cités de l'Amérique coloniale du XVIII ${ }^{\mathrm{e}}$ siècle (Hampton dans le New Hampshire, et Perquimans en Caroline du Nord) (Gallman, 1984), et il est d'environ deux ans en France des années 1790 aux années 1820 (Houdaille, 1982). Il en va de même dans les sociétés contemporaines. Les hommes nouvellement mariés sont en moyenne plus âgés que leurs épouses dans chacun des 91 pays et à chacune des périodes pour lesquelles des données sont disponibles des années 1900 aux années 1980 (sauf la Yougoslavie en 1945-1949, où l'écart d'âge moyen entre conjoints est de 0,1 an au profit des femmes) (Nations Unies, 1990). Les hommes nouvellement mariés sont aussi en moyenne plus âgés que leurs épouses dans 201 pays sur $202 \mathrm{au}$ cours des années 1990 et 2000 (l'exception étant San Marin, où l'écart d'âge moyen entre conjoints en 1995 est de 0,1 an au profit des femmes) (Nations Unies, 2000, 2007). Dans ces 202 pays, l'écart d'âge moyen entre nouveaux époux était de 3,5 ans au profit de l'homme, l'écart type de la distribution étant de 1,6 an.

En France en 1999 (donc, «en stock»), l'homme est plus âgé que sa conjointe dans deux couples sur trois, tandis que la femme n'est plus âgée que son conjoint que dans un couple sur cinq (un couple sur dix comprenant des conjoints nés la même année) (Barre et Vanderschelden, 2004) (1). Parmi les

(1) Cette répartition des écarts d'âge entre conjoints se retrouve, à l'identique, dans le Canada contemporain (Duchesne, 2004). 
mariages de célibataires célébrés en France en 2007 («en flux »), les mariages les plus fréquents sont ceux qui unissent une femme à un homme d'un an son aîné. Il est frappant de constater combien les mariages inverses dans lesquels c'est la femme qui a un an de plus que son époux - sont comparativement rares : ils sont moins fréquents que les mariages dans lesquels la femme a deux, trois ou même quatre ans de moins que son époux (Figure I).

\section{FIGURE I. - Nombre de mariages par écart d'âge au profit de l'homme entre nouveaux époux - France, 2007}

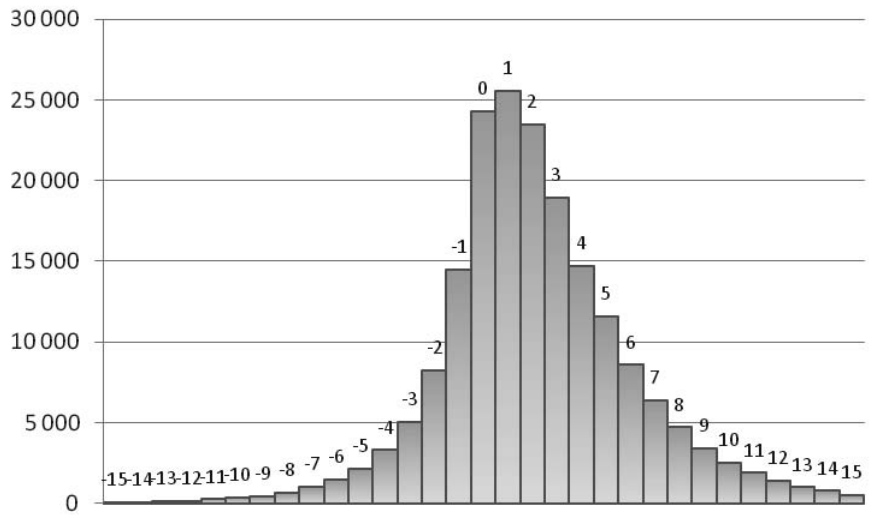

Champ : Les 188012 mariages entre célibataires en France métropolitaine en 2007 dont l'écart d'âge entre conjoints ne dépasse pas (en valeur absolue) quinze années.

Source : Insee, État-civil.

Si l'écart d'âge moyen entre conjoints au profit de l'homme est (presque) universellement positif, le degré auquel il est positif, lui, varie. À partir des données mises à disposition par les Nations Unies et à partir de la littérature empirique internationale sur le sujet, nous analysons ici les variations d'écart d'âge entre conjoints tout d'abord au niveau agrégé (en observant comment l'écart d'âge varie entre pays ainsi qu'au fil du temps), puis au niveau individuel (en observant comment l'écart d'âge varie entre couples d'une même société). Cela nous permettra d'établir de façon solide plusieurs observations, qu'il s'agira par la suite d'essayer d'expliquer.

\section{L'écart d'âge entre conjoints sur données agrégées : ses variations entre sociétés contemporaines, et au fil du temps}

Dans les années 1990 et 2000, alors que l'écart d'âge moyen entre nouveaux époux n'était que de 1,2 an en Irlande et 1,3 an au Vietnam, il était de 8,8 ans en Gambie et 9 ans en Guinée. De nos jours, les variations de l'écart d'âge moyen entre conjoints entre les pays du monde sont donc d'ampleur non négligeable. Mais quelles sont les caractéristiques dont les 
variations sont reliées à ces variations d'écart d'âge entre conjoints ? Pour le savoir, nous avons constitué à partir des statistiques mises à disposition par les Nations Unies une base de données recensant, pour tous les pays du monde pour lesquels des données sont disponibles, non seulement l'écart d'âge moyen entre nouveaux époux, mais aussi divers indicateurs sociodémographiques ou économiques (2).

Notons d'emblée que de telles données statistiques sont imparfaites : non seulement chacune des variables (notamment les âges moyens des femmes et des hommes au premier mariage) n'est pas renseignée dans chacun des 233 «pays ou entités » (3) recensés au total, mais, en outre, certaines variables ont été définies de façon légèrement différente selon les pays (et ce malgré les efforts d'harmonisation de l'ONU), et enfin la date exacte à laquelle elles ont été collectées n'est pas toujours la même (même si c'est le plus souvent une année entre 2000 et 2007, elle peut remonter jusqu'aux années 1990, sachant que les données datant des années 1990 sont plus souvent les seules à être disponibles pour les pays les moins développés). Estimant toutefois que de telles données sont trop précieuses - et irremplaçables - pour n'être pas exploitées, nous proposons de les analyser afin, tout au moins, d'en dégager quelques grandes tendances. Le tableau récapitulatif suivant liste ainsi les corrélations entre, d'une part, l'écart d'âge entre nouveaux époux et, d'autre part, divers indicateurs sociodémographiques et économiques (Tableau I).

\section{TABLEAU I. - Corrélations bivariées (coefficient de corrélation de Pearson) entre l'écart d'âge entre conjoints et diverses variables - tous les pays du monde, années 1990-2000}

\begin{tabular}{|c|c|c|c|}
\hline & & $\mathrm{N}$ & $\begin{array}{l}\text { Écart d'âge entre } \\
\text { conjoints au profit } \\
\text { de l'homme }\end{array}$ \\
\hline $\mathrm{a}$ & Âge moyen des hommes au $1^{\text {er }}$ mariage & 202 & $-0,247 * * *$ \\
\hline $\mathrm{b}$ & Âge moyen des femmes au $1^{\text {er }}$ mariage & 202 & $-0,642 * * *$ \\
\hline $\mathrm{c}$ & Taux brut de nuptialité & 101 & $-0,094$ \\
\hline $\mathrm{d}$ & Part d'hommes non célibataires à 45-49 ans & 191 & $0,252 * * *$ \\
\hline e & Part de femmes non célibataires à $45-49$ ans & 195 & $0,244 * *$ \\
\hline $\mathrm{f}$ & Écart entre les âges minima légaux au mariage & 141 & $0,395 * * *$ \\
\hline $\mathrm{g}$ & Part de moins de 15 ans dans la population & 197 & $0,581 * * *$ \\
\hline $\mathrm{h}$ & Part de 60 ans ou plus dans la population & 197 & $-0,433 * * *$ \\
\hline $\mathrm{i}$ & Espérance de scolarité masculine & 166 & $-0,582 * * *$ \\
\hline
\end{tabular}

(2) Ces données statistiques sont fournies sur le site Internet des Nations Unies (United Nations statistical databases) et proviennent de plusieurs sources: le Demographic yearbook system, la Millennium indicators database, les Social indicators, les Statistics and indicators on women and men, et l'enquête World contraceptive use 2005.
(3) Ces «pays ou entités» sont, pour la grande majorité d'entre eux, des États (dans l'ordre alphabétique, de l'Afghanistan au Zimbabwe), mais certains ne le sont pas, comme par exemple les départements d'outre-mer français, qui sont - pour une raison qui nous est inconnue - distincts de la métropole. 


\begin{tabular}{|c|c|c|c|}
\hline & & $\mathrm{N}$ & $\begin{array}{l}\text { Écart d'âge entre } \\
\text { conjoints au profit } \\
\text { de l'homme }\end{array}$ \\
\hline $\mathrm{j}$ & Espérance de scolarité féminine & 166 & $-0,627 * * *$ \\
\hline $\mathrm{k}$ & Taux d'activité masculin & 178 & $0,413 * * *$ \\
\hline 1 & Taux d'activité féminin & 178 & 0,013 \\
\hline $\mathrm{m}$ & Salaires féminins relativement aux salaires masculins & 63 & $-0,239$ \\
\hline $\mathrm{n}$ & Produit intérieur brut par tête & 190 & $-0,417 * * *$ \\
\hline o & Indicateur conjoncturel de fécondité & 193 & $0,650 * * *$ \\
\hline $\mathrm{p}$ & Taux de prévalence des méthodes de contraception & 165 & $-0,661 * * *$ \\
\hline
\end{tabular}

Source : Nations Unies.

Note : $*<0,05 ; * *<0,01 ; * * *<0,001$.

L'écart d'âge moyen entre conjoints au profit de l'homme tend à être relativement élevé dans les pays dans lesquels les hommes et, plus encore, les femmes se marient relativement tôt $(a$ et b) et se marient relativement fréquemment ( $\mathrm{d}$ et e), et dans lesquels les femmes sont relativement fécondes (o) et utilisent relativement peu la contraception (p). Ce sont aussi des pays relativement jeunes ( $g$ et $h$ ) et pauvres $(n)$, dans lesquels les hommes et plus encore les femmes sont relativement peu scolarisés ( $i$ et j) (4). Même si ce descriptif n'est pas parfaitement univoque (l'écart d'âge entre conjoints n'est pas lié de façon significative au taux brut de nuptialité, au taux d'activité féminin, ni au niveau relatif de salaire des femmes), il apparaît de façon assez claire que le développement socioéconomique et ses corrélats - scolarisation, enrichissement, report du mariage, baisse de la fécondité, vieillissement, etc. - vont de pair avec des écarts d'âge entre conjoints réduits (5). Dans une étude concernant 57 pays du monde autour de 1960, on avait déjà observé que «plus les épouses sont jeunes à leur mariage plus la différence d'âge avec leur époux est grande »(Dixon, 1971, p. 216), et des résultats analogues ont aussi été répliqués sur des données récentes (Saardchom, 2001).

Si l'on étudie non plus les variations de l'écart d'âge entre conjoints entre pays, mais les évolutions de l'écart d'âge entre conjoints au fil du temps, qu'observe-t-on ? En France, «l'écart entre hommes et femmes tend à se réduire au fil des générations » (Barre et Vanderschelden, 2004, p. 22) : l'écart d'âge entre conjoints passe de 2,8 ans en moyenne pour les couples formés dans les années 1950 à 2,3 ans pour ceux formés dans les années 1990 (Vanderschelden, 2006). Alors que les couples dont l'homme a plus d'un an que sa femme constituaient $62 \%$ des couples formés dans les années 1950, ils

(4) Le fait que l'écart d'âge entre conjoints tende à décroître avec le niveau d'instruction des femmes avait déjà été repéré dans une analyse portant sur les couples (mariés ou non) formés entre 1974 et 1984 dans 28 pays en développement d'Asie, d'Afrique et d'Amérique (Casterline, Williams et McDonald, 1986).

(5) Cette observation réalisée entre pays concorde avec l'observation réalisée entre régions françaises au XIX ${ }^{\mathrm{e}}$ siècle. En effet, en France, à cette époque, l'écart d'âge entre conjoints au profit de l'homme est plus élevé dans le Sud - relativement pauvre et peu industrialisé - qu'il ne l'est dans le Nord (Le Bras et Todd, 1981, p. 183 ; Bonneuil, 1992). 
ne constituent plus «que » $54 \%$ des couples formés dans les années 1990 (ibid.). En France donc, au fil de la seconde moitié du $\mathrm{XX}^{\mathrm{e}}$ siècle, l'écart d'âge entre conjoints a eu tendance à baisser (6).

C'est ce que l'on observe plus généralement en Europe occidentale, où l'écart d'âge entre conjoints est passé de 3,7 ans dans les années 1950 à 3,5 ans dans les années 1960, 3,2 ans dans les années 1970, 2,9 ans dans les années 1980 et 2,8 ans dans les années 1990 (Nations Unies, 1990, 2007). Sur plus long terme, l'écart d'âge entre nouveaux époux est passé aux Pays-Bas de 4,5 ans dans les années 1850 à 2,6 ans dans les années 1970 (avant de se stabiliser jusqu'aux années 1990) (Van Poppel et al., 2001). Sur les quatre autres continents, l'écart d'âge a eu tendance à baisser des années $1950 \mathrm{ou}$ 1960 aux années 1990 dans près de $90 \%$ des pays (64 pays sur 72 pour lesquels des données sont disponibles) (Nations Unies, 1990). En Afrique, plus particulièrement, on observe «un resserrement de l'écart d'âge entre conjoints dans la quasi-totalité des pays » (Hertrich et Pilon, 1997, p. 2) ; sur 32 pays analysés entre les années 1960 et 1990, seuls trois ont connu un accroissement de l'écart d'âge entre conjoints (ibid.) (7).

À un niveau agrégé, on observe donc que l'écart d'âge entre conjoints au profit de l'homme tend à être plus élevé dans les sociétés moins développées, et à décroître au fil du temps dans la plupart des pays du monde. Voyons maintenant comment varie l'écart d'âge non plus entre sociétés ou au fil du temps, mais entre couples d'une même société.

\section{L'écart d'âge entre conjoints sur données individuelles : ses variations selon l'âge au mariage de chaque conjoint}

Si la littérature empirique internationale sur l'écart d'âge entre conjoints a repéré une association statistique forte entre ce dernier et une autre variable, c'est bien avec l'âge au mariage de chaque conjoint : à notre connaissance, dans toutes les sociétés humaines qui ont fait l'objet d'investigations sur ce point, l'écart d'âge entre conjoints au profit de l'homme croît avec l'âge de l'homme à la mise en couple et décroît avec l'âge de la femme à la mise en couple (8). En d'autres termes, les hommes qui se mettent en couple plus tardivement le font avec une femme relativement plus jeune qu'eux, alors que

(6) Notons que cette réduction de l'écart d'âge moyen entre conjoints au fil du temps peut avoir des conséquences dans des domaines relativement inattendus. Par exemple, on a montré que la baisse de l'écart d'âge entre conjoints aux États-Unis entre les générations 1900 et 1955 , en réduisant le risque de veuvage pour les femmes et en accroissant celui des hommes, a réduit la propension des femmes et accru celle des hommes à fréquenter les maisons de retraite (Lakdawalla et Schoeni, 2003).

(7) Par exemple, l'écart d'âge moyen entre nouveaux époux est passé de 5,6 ans en 1966 à 3,7 ans en 1998 en Algérie (Ouadah-Bedidi et Vallin, 2003), et de 10 ans en 1967 à 5,5 ans en 2001 à Kinshasa (Kayiba Mbelu, 2005).

(8) Ce phénomène observé au niveau individuel diffère donc du phénomène observé au niveau agrégé, puisqu'entre pays dans les années 1990 et 2000 l'écart d'âge moyen entre époux décroît non seulement avec l'âge moyen des femmes au mariage, mais aussi - moins fortement - avec l'âge moyen des hommes au mariage (Tableau I). 
les femmes qui se mettent en couple plus tardivement le font avec un homme relativement moins âgé qu'elles. On a parfois suggéré que plus les individus se mettent en couple tard, plus les autres membres de leur génération se sont déjà mis en couple, ce qui les conduirait à rechercher leur conjoint dans d'autres groupes d'âge que le leur; toutefois, cela ne saurait expliquer les variations d'écart d'âge selon l'âge à la mise en couple, puisque l'on se demande pourquoi les hommes recruteraient leurs conjointes dans des groupes d'âge de plus en plus jeunes par rapport au leur, alors que dans la même situation les femmes recruteraient leurs conjoints dans des groupes d'âge de plus en plus proches du leur.

Observons précisément l'écart d'âge moyen entre époux selon l'âge des époux au mariage, parmi les individus qui se sont mariés pour la première fois en France en 2007 (Figure II).

\section{FIGURE II. - Écart d'âge moyen entre époux selon l'âge des époux au mariage - France, 2007}

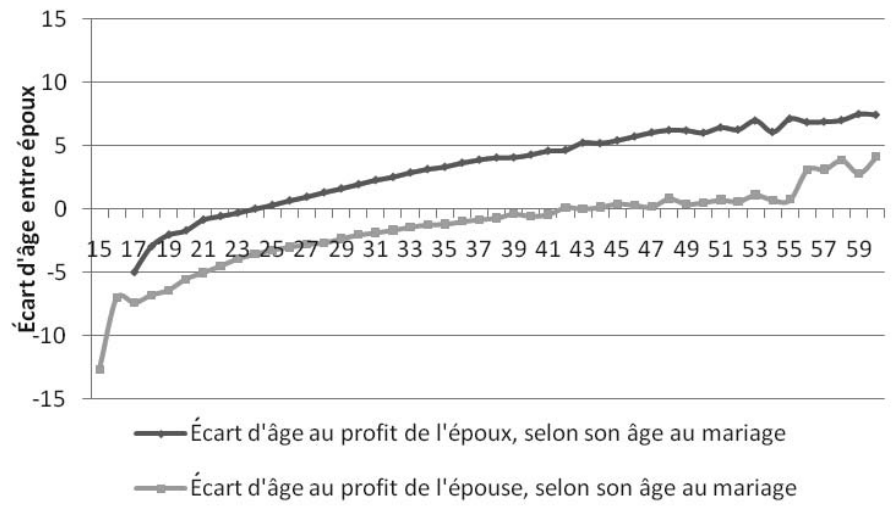

Champ : Les 189809 mariages entre célibataires en France métropolitaine en 2007. Source : Insee, État-civil.

Sur ce graphique, plusieurs phénomènes sont repérables. Tout d'abord, les deux courbes sont croissantes (plutôt que d'être stables ou décroissantes), ce qui signifie que plus un individu (homme ou femme) se marie à un âge tardif (en abscisses), plus il a de chances d'être relativement plus âgé ou relativement moins jeune que son époux/se. Cela correspond au fait qu'il existe une corrélation positive entre les âges des époux : en matière d'âges, «qui se ressemble s'assemble » (9). Une part de ce phénomène pourrait s'expliquer

(9) Cette observation a déjà été réalisée dans chacun des 28 pays en développement d'Asie, d'Afrique et d'Amérique dans lesquels étaient analysés les couples (mariés ou non) formés entre 1974 et 1984 (même en contrôlant les niveaux de diplôme des conjoints) (Casterline, Williams et McDonald, 1986). 
par un pur effet probabiliste : plus on avance en âge, plus le stock de conjoints plus jeunes disponibles croît au détriment du stock de conjoints plus âgés disponibles, accroissant ainsi la probabilité de se mettre en couple avec un conjoint plus jeune, plutôt que plus âgé.

Ensuite, si l'on considère non plus la direction des courbes mais leur niveau, il apparaît que la courbe des hommes dépasse la valeur zéro (aucun écart d'âge) dès qu'ils ont atteint 24 ans (soit bien avant l'âge moyen des hommes au premier mariage en 2007, qui est de 31,4 ans), alors que la courbe des femmes ne la dépasse qu'à 42 ans (soit bien après l'âge moyen des femmes au premier mariage en 2007, qui est de 29,5 ans). Aux âges de plus forte nuptialité, donc, les hommes sont en moyenne plus âgés que leur épouse, et les femmes sont en moyenne moins âgées que leur époux. Au total, l'écart d'âge moyen entre nouveaux époux en France en 2007 est de 2,22 ans, au profit de l'homme.

Enfin - et c'est le point qui importe ici le plus -, à partir de 24 ans, la courbe des hommes dépasse la valeur zéro de plus en plus (plutôt que de se maintenir à un même niveau), et de même jusqu'à 41 ans la courbe des femmes se rapproche de zéro de plus en plus. Cela signifie que plus un homme se marie tard à partir de 24 ans plus il est relativement plus âgé que son épouse (plus l'écart d'âge se creuse), alors que plus une femme se marie tard jusqu'à 42 ans moins elle est relativement plus jeune que son époux (plus l'écart d'âge se comble). On observe donc bien que, aux âges de plus forte nuptialité, plus les hommes se marient tard plus l'écart d'âge avec leur épouse se creuse, alors que plus les femmes se marient tard plus l'écart d'âge avec leur époux se comble. Ce phénomène avait déjà été observé à Paris dans les promotions de mariage 1936, 1956, 1960 et 1964 (Vallot, 1971), et plus généralement en France dans les premières unions de 1984 (Bozon, 1991), dans les premières unions formées entre 1968 et 1985 (Bozon, 1990a, 1990b) et dans les unions formées des années 1930 aux années 1990 (Vanderschelden, 2006) (10).

Comme nous l'avons dit, un tel phénomène a été observé dans des sociétés de diverses époques et de plusieurs continents. Pour ce qui concerne des périodes historiques, "l'écart d'âge entre époux apparaît d'autant plus élevé que la mariée est plus jeune » au Canada au XVII siècle (Charbonneau, 1980, p. 1104), et l'écart d'âge entre époux est d'autant plus élevé que l'homme est plus âgé lors de son premier mariage dans deux cités de l'Amérique coloniale

(10) Il est intéressant de constater que ce phénomène général est vérifié aussi dans les unions relativement atypiques que sont, dans la France du $\mathrm{XX}^{\mathrm{e}}$ siècle, les mariages entre apparentés. Parmi les unions qui ont nécessité une dispense civile au mariage pour motif que les époux étaient consanguins - unions qui sont presque totalement des unions entre oncle et nièce ou tante et neveu -, des oncles épousent environ douze fois plus souvent leur nièce que des tantes n'épousent leur neveu en 1930-1935 (Sutter et Lévy, 1959) (et encore, dans les unions entre tante et neveu des années 1930-1932 et 1946-1957 la femme n'est plus âgée que son mari que de 2,8 ans en moyenne) (ibid.), et des oncles épousent environ sept fois plus souvent leur nièce que des tantes n'épousent leur neveu en 1960-1992 (Biégelmann-Massari, 1996a, 1996b). 
du XVIII ${ }^{\mathrm{e}}$ siècle (Gallman, 1984). Analysant les mariages de la première moitié du $\mathrm{XX}^{\mathrm{e}}$ siècle dans un village de l'île grecque de Karpathos, dans le Dodécanèse, un chercheur note aussi : «Étant donné que le vieillissement biologique a des effets différents sur la valeur matrimoniale des deux sexes, la différence d'âge entre époux variait [...] selon l'âge au mariage de l'homme. Elle était d'autant plus importante que ce dernier était plus âgé : deux ans pour les hommes de 21 à 25 ans et jusqu'à vingt ans pour les hommes de 51 à 60 ans. »(Vernier, 1985, p. 8).

Concernant des sociétés contemporaines, on a observé qu'en République fédérale d'Allemagne, dans les générations 1932-1933, «à mesure qu'ils avancent en âge, les hommes choisissent leur partenaire dans un éventail élargi et épousent des femmes qui sont, par rapport à eux, de plus en plus jeunes » (Festy, 1971, p. 339). On a aussi observé que, dans 18 pays d'Afrique subsaharienne des années 1990, l'écart d'âge entre conjoints est plus faible si la femme en est à sa seconde union ou, plus encore, à sa énième union, plutôt qu'à sa première union (Barbieri et Hertrich, 2005). Il semble en aller de même en Algérie en 1998, puisque « les écarts entre conjoints sont les plus faibles là [dans les régions administratives] où l'âge moyen au premier mariage des femmes est le plus élevé » (Ouadah-Bedidi et Vallin, 2003, p. 297). L'écart d'âge entre nouveaux époux est aussi d'autant plus prononcé que l'homme se marie plus tard et que la femme se marie plus tôt au Brésil en 1996 (Otta et al., 1999), ainsi qu'à Haïti en l'an 2000 (Cayemittes et al., 2001).

Enfin, notons que la pratique selon laquelle les hommes se mettent en couple avec des femmes d'autant plus jeunes qu'eux qu'ils sont eux-mêmes plus âgés lors de leur mise en couple est parfois explicitement désirée et recommandée. Comme on l'a constaté en France à partir des déclarations d'individus mis en couple entre 1968 et 1985, « ce sont bien les femmes les plus jeunes qui souhaitent l'écart le plus important avec leur conjoint alors que les femmes plus âgées aimeraient une différence plus réduite. Pour les hommes, l'évolution est strictement inverse : aux âges jeunes, ce sont des femmes proches par l'âge qui sont recherchées, et des femmes sensiblement plus jeunes aux âges tardifs » (Bozon, 1990a, p. 349). Par ailleurs, si les individus respectaient les prescriptions formulées par certains proverbes «l'homme doit prendre une femme qui ait la moitié de son âge, plus sept ans » (proverbe chinois), ou l'épouse doit avoir « autant de fois sept ans qu'il (l'époux) en a de fois neuf » (proverbe d'Ancien Régime cité dans Bologne, 1995) -, l'écart d'âge entre conjoints au profit de l'homme croîtrait effectivement avec l'âge de l'homme au mariage (Figure III). 


\section{FIGURE III. - Écart d'âge « idéal » entre époux par âge de l'homme au mariage}

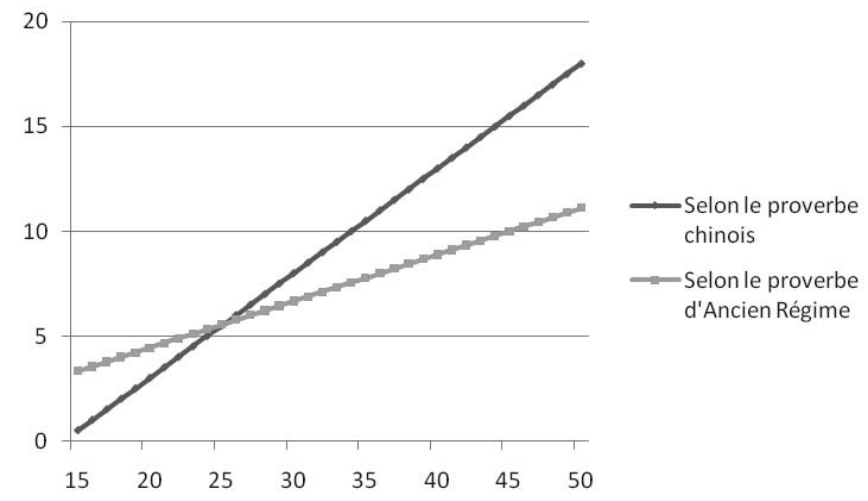

À un niveau individuel, on observe donc que l'écart d'âge entre conjoints au profit de l'homme (en pratique, mais aussi apparemment dans l'ordre des préférences) tend à croître avec l'âge de l'homme à la mise en couple et à décroître avec l'âge de la femme à la mise en couple. Voyons maintenant comment varie l'écart d'âge non plus selon les âges des conjoints à la mise en couple, mais selon leur position sociale.

\section{L'écart d'âge entre conjoints sur données individuelles : ses variations selon la position sociale du couple}

La littérature empirique internationale indique que l'écart d'âge entre conjoints au profit de l'homme tend à être d'autant plus élevé que l'homme détient une position sociale plus élevée et, plus généralement, qu'il a plus de valeur sur le marché matrimonial. En d'autres termes, plus les hommes sont relativement dotés d'argent, de prestige et/ou de pouvoir, plus leur conjointe tend à être plus jeune qu'eux.

Ce phénomène a été observé à plusieurs reprises dans la France d'Ancien Régime et au XIX siècle. Par exemple, dans un village normand (Tourouvre-au-Perche), aux XVII et XVIII siècles, l'écart d'âge moyen entre conjoints s'élève avec la position sociale : il avoisine zéro pour les sabotiers, un peu plus d'un an pour les manœuvres, plus de deux ans pour les artisans, et plus de trois ans pour les marchands et laboureurs (Charbonneau, 1970). En France, au XIX ${ }^{\mathrm{e}}$ siècle, les écarts d'âge «sont élevés pour les propriétaires (6,3 ans), plus faibles pour les cultivateurs et les laboureurs (entre 3,8 et 4,5), encore plus faibles (entre 2,5 et 3,0) pour les domestiques, journaliers, maçons, manœuvres et manouvriers indistinctement » (Bonneuil, 
1992, p. 97) (11). Commentant les résultats d'une analyse portant sur les mariages dans la bourgeoisie parisienne de 1789 à 1804, un auteur note ainsi : «Nous découvrons là un phénomène extrêmement intéressant: quand le niveau économique et social s'abaisse, il y a de plus en plus de femmes plus âgées que leurs maris. [...] Il semble donc que plus on descendait dans l'échelle sociale, plus l'âge au mariage des filles reculait, l'âge des hommes ne semblant jouer aucun rôle. » (Duplessis-Le Guélinel, 1954, p. 25) (12).

Comme on le voit (Figure IV), en France l'écart d'âge entre conjoints est effectivement sensiblement supérieur dans les classes aisées à ce qu'il est dans la population générale, au moins jusqu'à la seconde moitié du $\mathrm{XX}^{\mathrm{e}}$ siècle.

\section{FIGURE IV. - Écart d'âge moyen entre époux au profit de l'homme selon la position sociale du couple - France, 1600-2000}

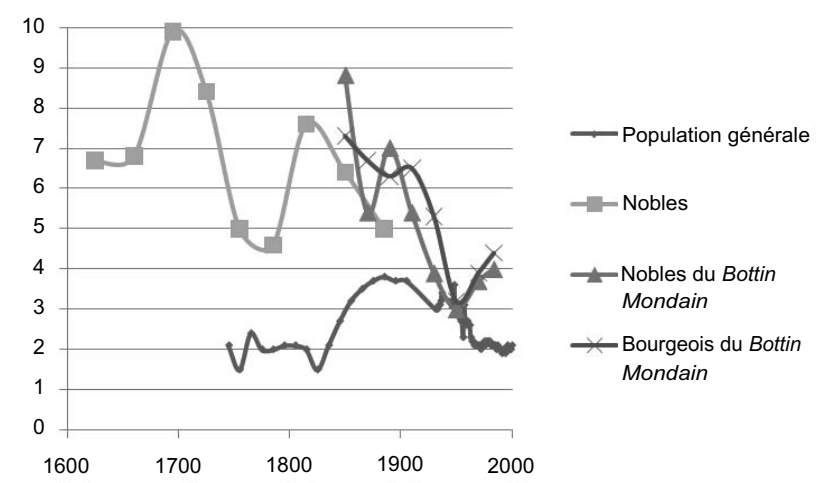

Sources: Henry et Houdaille (1979) (population générale de 1670 à 1909), Lery (1975) (population générale de 1931 à 1938), Sardon (2005) (population générale de 1946 à 2000), Houdaille (1989) (nobles de 1600 à 1899), Grange (1996) (nobles et bourgeois du Bottin Mondain de 1840 à 1987).

(11) Cette observation réalisée au niveau national est confirmée par plusieurs monographies locales. Par exemple, à Bordeaux, au $\mathrm{XIX}^{\mathrm{e}}$ siècle, « les écarts d'âge sont constamment plus importants dans la bourgeoisie que dans le peuple » (Guillaume, 1972, p. 109). De même, à Orléans, parmi les premiers mariages de l'année 1911, l'écart d'âge entre conjoints (qui est en moyenne de 2 ans et 10 mois) est de 6 ans et 4 mois lorsque le mari est un bourgeois, et de 1 an et 3 mois chez les ouvriers des métaux : «Il est très rare, dans la bourgeoisie, que la femme soit plus âgée que le mari, ou même qu'elle ait son âge, alors que, dans les classes moyennes, on trouve $15 \%$ de femmes plus âgées, et $25 \%$ chez les ouvriers. En revanche, parmi ceux-ci, on trouve $25,7 \%$ de maris plus âgés que leur femme d'au moins 5 ans, contre $33,4 \%$ dans les classes moyennes et $68 \%$ dans la bourgeoisie. » (Prost, 1981, p. 692).

(12) Dans une analyse des âges au mariage des aristocrates français du XVII ${ }^{\mathrm{e}}$ jusqu'au $\mathrm{XIX}^{\mathrm{e}}$ siècle, un chercheur indique aussi que « comme dans toutes les études portant sur des groupes de privilégiés (bourgeois ou nobles), l'écart d'âge entre époux est plus grand que dans l'ensemble de la population » (Houdaille, 1989, p. 508). En effet, au sommet de la hiérarchie sociale européenne du XVIII ${ }^{\mathrm{e}}$ siècle (dans les mariages contractés par les familles régnantes d'Europe, les familles ducales anglaises, ou les vieilles familles de la classe dirigeante de Genève), l'écart d'âge entre conjoints est plus élevé que dans la population générale (Lévy et Henry, 1960). 
Une étude monographique portant sur un village de l'île grecque de Karpathos, dans le Dodécanèse, analyse avec finesse les variations d'écart d'âge entre conjoints selon la position sociale des époux (Vernier, 1977, 1985). Dans ce village, "sous le rapport économique, [les hommes] avaient d'autant plus de valeur qu'ils étaient plus âgés et donc, en principe, plus riches. Aussi [de 1915 à 1980] c'était lorsque l'on avait de 26 à 30 ans et que l'on combinait harmonieusement, si l'on peut dire, l'argent et la jeunesse que l'on pouvait prendre les femmes les plus jeunes (20,6 ans), et quand on avait de 31 à 35 ans, on pouvait épouser des femmes aussi jeunes (21,3 ans) que celles des hommes de 16 à 20 ans. Ceux qui se mariaient les plus jeunes et qui n'avaient donc pas eu le temps d'économiser étaient obligés d'épouser des femmes qui, en moyenne, étaient plus âgées qu'eux. Et c'était encore plus vrai quand ces hommes étaient des cadets et qu'ils ne disposaient donc pas même d'un héritage : ils épousaient des femmes qui, âgées de 22 ans en moyenne, étaient de quatre années plus vieilles qu'eux » (Vernier, 1985, p. 8). De façon extrême, «le villageois qui présente avec sa femme l'écart d'âge le plus spectaculaire cumule les handicaps du cadet et de l'enfant naturel. Peu soucieux d'un capital symbolique de toute façon inexistant, il a épousé à 21 ans une femme de 40 ans qui, elle-même, du fait de son âge, était condamnée à choisir parmi tous les jeunes du village le parti le plus mauvais socialement » (ibid., p. 22).

À un niveau individuel, on observe donc que, dans les sociétés agraires au moins, l'écart d'âge entre conjoints au profit de l'homme tend à croître avec la position sociale de ce dernier.

\section{Bilan des observations}

Les investigations empiriques que nous avons menées à propos de l'écart d'âge entre conjoints nous conduisent à plusieurs constats : outre que l'écart d'âge entre conjoints au profit de l'homme semble exister dans la (quasi-) totalité des sociétés humaines connues, on observe les phénomènes suivants :

- l'écart d'âge est plus élevé dans les sociétés moins développées du point de vue socioéconomique ;

- l'écart d'âge tend à baisser depuis les années 1950 dans la plupart des pays du monde ;

- l'écart d'âge croît avec l'âge de l'homme à la mise en couple et décroît avec l'âge de la femme à la mise en couple ;

- l'écart d'âge croît avec la position sociale de l'homme, tout au moins dans les sociétés agraires.

La question qui se pose, dès lors, est de savoir pourquoi l'on observe ces phénomènes plutôt que d'autres. 


\section{Un modèle explicatif de l'écart d'âge entre conjoints et de ses variations}

$\mathrm{Si}$, de façon universelle, les hommes se mettent en couple avec des femmes qui sont, en moyenne, plus jeunes qu'eux, c'est vraisemblablement parce que les membres de l'un des deux sexes au moins préfèrent de tels appariements à des appariements entre individus de mêmes âges (ou a fortiori à des appariements présentant un écart d'âge entre conjoints au profit de la femme). Effectivement, selon l'enquête menée en 2005 par l'IFOP pour le site de rencontres «Parship » auprès des internautes adultes et célibataires, même si $56 \%$ des répondants affirment que l'âge du partenaire n'est pas un critère important et même si $10 \%$ des répondants affirment que le partenaire doit avoir le même âge que le leur, $36 \%$ des femmes contre seulement $5 \%$ des hommes estiment que le partenaire doit «être plus vieux que vous (mais pas plus de 10 ans) », et $23 \%$ des hommes contre seulement $4 \%$ des femmes estiment que le partenaire doit être «plus jeune que vous (mais pas plus de 10 ans)». De façon plus générale, dans un échantillon de plus de 10000 individus issus de 37 sociétés (issues de 33 États et situées sur tous les continents, dont cinq d'entre elles sur des îles), on a observé que dans 37 sociétés sur 37 les hommes déclarent préférer des conjointes plus jeunes qu'eux (différence significative au seuil de 0,0001 dans chacune des 37 sociétés) et les femmes déclarent préférer des conjoints plus âgés qu'elles; ces préférences étaient d'ailleurs largement traduites dans les pratiques puisque, dans les sociétés pour lesquelles des données sont disponibles, on observe un écart d'âge entre conjoints proche des écarts d'âge préférés par les femmes et les hommes (Buss, 1989). Il apparaît donc que l'écart d'âge entre conjoints, loin d'être issu d'un simple " consentement », comme le suggère un article sur le sujet (Bozon, 1990a, 1990b), dérive d'une préférence, partagée en outre par les membres des deux sexes (13).

La question, dès lors, consiste à comprendre pourquoi les hommes et les femmes préfèrent se mettre en couple de telle façon que l'homme soit plus âgé que la femme. Par exemple, affirmer qu'il existe une domination masculine sur le marché sexuel et matrimonial, sur lequel les hommes peuvent affirmer leur préférence pour des femmes plus jeunes, ne peut pas être considéré comme une explication adéquate du phénomène car - outre que cette affirmation omet de préciser que les femmes préfèrent elles aussi un écart d'âge au profit de l'homme et contribuent activement à le produire - cela ne permet pas de comprendre pourquoi les hommes, eux, préféreraient se mettre en couple avec des femmes plus jeunes (plutôt que de même âge ou plus âgées).

Pour expliquer l'existence - le caractère universel - de l'écart d'âge moyen entre conjoints au profit de l'homme ainsi que les variations de l'écart d'âge

(13) Ce fait semble par ailleurs admis dans l'article même auquel nous faisons ici référence, puisqu'il mentionne un «désir d'être dominées », un « goût pour les hommes mûrs », et un « refus de l'homme plus jeune» (c'est nous qui soulignons). 
entre sociétés et entre couples, deux chercheurs ont proposé un modèle explicatif issu de la théorie du choix rationnel (Bergstrom et Bagnoli, 1993). Présentons ici ce modèle, avant d'en évaluer le pouvoir explicatif et les éventuelles limites (14). Selon ce modèle de l'écart d'âge entre conjoints, les individus - rationnels - choisiraient leur propre âge au mariage de façon à pouvoir s'apparier avec le conjoint le plus désirable possible. En d'autres termes, hommes et femmes se marieraient aux âges auxquels ils connaissent leurs «pics de désirabilité » respectifs (aux yeux des membres du sexe opposé), et c'est l'écart entre les âges auxquels hommes et femmes connaîtraient leurs pics de désirabilité respectifs qui inciterait les premiers à reporter leur mariage plus longtemps que les secondes, conduisant ainsi les couples à connaître un écart d'âge moyen entre conjoints au profit de l'homme.

Plus précisément, ce modèle de l'écart d'âge entre conjoints repose sur plusieurs hypothèses simplificatrices, qu'il convient d'expliciter. Tout d'abord, ce modèle suppose que les membres de chaque sexe ont le même ordre de préférences concernant les membres du sexe opposé (les hommes s'accordent tous sur la hiérarchie de désirabilité des femmes, et de même les femmes s'accordent toutes sur la hiérarchie de désirabilité des hommes), et que les individus se mettent en couple selon leur rang de désirabilité (seul l'homme le plus désirable parvient à se marier avec la femme la plus désirable, seul le deuxième homme le plus désirable parvient à se marier avec la deuxième femme la plus désirable, etc.). Ensuite, ce modèle suppose que les individus ne peuvent se mettre en couple qu'à deux âges - «l'âge précoce » et «l'âge tardif »-, et que le report de la mise en couple jusqu'à l'âge tardif engendre un coût puisqu'il prive l'individu qui l'effectue des gains (matériels et immatériels) de l'union dont il aurait pu bénéficier en se mettant en couple dès l'âge précoce. Enfin - et c'est là l'hypothèse majeure -, ce modèle suppose que le degré de désirabilité d'une femme est connu des hommes dès qu'elle a atteint l'âge précoce, tandis que le degré de désirabilité des hommes

(14) D'autres modèles explicatifs de l'écart d'âge entre conjoints inspirés de la théorie du choix rationnel ont été avancés par divers chercheurs, mais nous proposons de n'en détailler ici qu'un seul, qui est le plus explicite. Par souci de complétude, notons toutefois que, selon les modèles alternatifs les plus simples (Keeley, 1977 ; Oppenheimer, 1988), l'existence d'un écart d'âge moyen entre conjoints au profit de l'homme s'expliquerait par le fait que les coûts du report du mariage (search costs) sont plus élevés pour les femmes que pour les hommes, en raison du double fait qu'au fil des âges les femmes perdent plus de leur valeur aux yeux des hommes que les hommes n'en perdent aux yeux des femmes - si bien que le vieillissement coûte aux femmes en termes de qualité du conjoint qu'elles peuvent obtenir - et qu'au fil des âges les femmes perdent plus de leur fertilité que les hommes - si bien que le vieillis- sement coûte aux femmes en termes de fécondité potentielle. Selon d'autres modèles, relativement proches (Cohen, 1987, 2002; Grossbard-Shechtman, 1993), l'existence d'un écart d'âge moyen entre conjoints au profit de l'homme s'expliquerait par le souci qu'auraient les femmes d'éviter une situation d'aléa moral (moral hazard) qui leur est défavorable : en se mettant en couple avec un homme plus âgé qu'elles, les femmes chercheraient à éviter la situation dans laquelle elles s'investiraient dans leur couple (à travers l'éducation des enfants) avant que leur conjoint en fasse de même (à travers le partage de ses salaires élevés de fin de carrière), ce qui pourrait tenter ce dernier de profiter des investissements de sa conjointe pour ensuite la quitter et, désormais doté de salaires élevés, se remettre en couple avec une nouvelle conjointe dont il profiterait à nouveau des investissements dans son couple. 
n'est parfois révélé aux femmes qu'à partir du moment où ils atteignent l'âge tardif (15).

Si l'on peut admettre que les deux premières séries d'hypothèses sont nécessaires à la construction de tout modèle explicatif de l'écart d'âge, qu'est-ce qui pourrait justifier la troisième ? Pourquoi la désirabilité des femmes serait-elle révélée plus précocement aux hommes que celle des hommes ne l'est aux femmes ? Selon les auteurs, la raison en serait la suivante. Si, d'un côté, les hommes valorisent particulièrement les femmes pour leur beauté physique ainsi que pour leur degré de fertilité actuel et la durée restante de leur période fertile, le pic de désirabilité des femmes aux yeux des hommes serait atteint par les femmes - et connu par les hommes - dès un âge relativement précoce. Si, d'un autre côté, les femmes valorisent particulièrement les hommes pour le niveau ainsi que la prévisibilité du niveau de ressources socioéconomiques qu'ils parviendront à posséder, le pic de désirabilité des hommes aux yeux des femmes ne serait atteint par les hommes - et connu par les femmes - qu'à partir d'un âge relativement tardif (dans les sociétés agraires, une fois seulement qu'ils ont accumulé un capital suffisant pour acheter des terres, et, dans les sociétés industrielles puis tertiaires, une fois qu'ils ont terminé leurs études et/ou sont entrés sur le marché du travail, voire y ont obtenu des promotions). En conséquence, les femmes, sachant qu'elles sont particulièrement valorisées à des âges relativement précoces, auraient tout intérêt à ne pas reporter leur mariage trop longtemps, tandis que les hommes, sachant eux aussi qu'ils sont particulièrement valorisés à des âges relativement tardifs, pourraient avoir intérêt à reporter leur mariage en vue de pouvoir s'apparier avec une femme plus désirable à leurs yeux que celle qu'ils auraient pu obtenir à un âge précoce (16). Certains hommes se mettant alors en couple plus tardivement que les femmes, on observerait bel et bien un écart d'âge moyen entre conjoints au profit de l'homme.

Notons ici que ces hypothèses - selon lesquelles les hommes accorderaient plus d'importance que les femmes aux caractéristiques physiques de leur conjointe et les femmes accorderaient plus d'importance que les hommes à la position sociale de leur conjoint - sont largement validées par la littérature empirique sur les goûts et pratiques en matière de choix du conjoint. Par exemple, dans des petites annonces matrimoniales françaises de la fin des années 1970, les hommes qui apparaissaient relativement désirables du point de vue physique ou socioéconomique en profitaient pour accroître leurs exigences physiques, tandis que les femmes qui apparaissaient relativement

(15) En d'autres termes, ce modèle suppose que les hommes disposent d'une information parfaite sur les caractéristiques de chaque femme dès qu'elle a atteint l'âge précoce, alors que les femmes ne disposent que d'une information imparfaite sur les caractéristiques de chaque homme tant qu'il n'a pas atteint l'âge tardif.

(16) Comme on l'a dit, « la probabilité pour une femme de former une première union décroît rapidement avec l'âge, en raison notamment de la concurrence des femmes plus jeunes. [...] À trop temporiser, une femme court de lourds risques !» (Bozon, 1990a, p. 333). Par contraste, « à mesure qu'il s'éloigne de sa $20^{\mathrm{e}}$ année, le candidat à la vie conjugale voit s'élargir sensiblement l'éventail des âges des compagnes potentielles » (ibid.). 
désirables du point de vue physique ou socioéconomique en profitaient pour accroître leurs exigences socioéconomiques (Singly, 1984). De façon plus générale, dans un échantillon de plus de 10000 individus issus de 37 sociétés, les hommes, plus que les femmes, valorisent chez leurs partenaires potentielles les indicateurs physiques de fertilité, et les femmes, plus que les hommes, valorisent chez leurs partenaires potentiels les indicateurs d'acquisition de ressources (Buss, 1989). D'une part, dans 37 sociétés sur 37, les hommes accordent plus d'importance à l'attractivité physique que les femmes (cette différence étant significative au seuil de 0,05 dans 34 sociétés). D'autre part, dans 36 sociétés sur 37, les femmes valorisent plus que les hommes les perspectives financières qu'apporte un conjoint (la seule exception étant l'Espagne, où l'effet observé est dans le sens attendu mais n'est pas significatif), et, dans 29 sociétés sur 37, les femmes valorisent (significativement) plus que les hommes l'ambition et l'ardeur au travail (la seule société dans laquelle ce sont les hommes qui valorisent significativement plus l'ambition et l'ardeur au travail étant la société zoulou, dans laquelle c'est la femme qui construit la maison, va chercher l'eau et exerce d'autres tâches exigeantes physiquement) (ibid.).

Comme on pouvait s'en douter, hommes et femmes sont d'ailleurs parfaitement conscients des caractéristiques que les membres de l'autre sexe survalorisent chez eux; par exemple, en France, dans les petites annonces matrimoniales de la fin des années 1970, les hommes mentionnaient leurs qualités socioéconomiques plus que les femmes et les femmes mentionnaient leurs qualités physiques plus que les hommes (Singly, 1984). Ainsi, on peut admettre - avec le modèle présenté ici - que les femmes anticipant une relative dévalorisation au fil des âges ont conscience du fait qu'elles ont peu intérêt à reporter leur mise en couple, tandis que les hommes anticipant une ascension professionnelle ont conscience du fait qu'ils peuvent avoir intérêt à reporter leur mise en couple jusqu'au moment où ils atteignent une position sociale qui les valorisera aux yeux des femmes.

L'importance relative qu'accordent les femmes à la position sociale de leur conjoint et celle qu'accordent les hommes à la jeunesse de leur conjointe ont d'ailleurs plusieurs conséquences sociodémographiques bien connues. D'une part, le risque de célibat définitif tend à baisser à mesure que s'élève la position sociale des hommes (mesurée par leur niveau de diplôme), ce qui n'est pas le cas, bien au contraire, pour les femmes (Robert-Bobée et Mazuy, 2005). D'autre part, les taux de mariage puis de remariage baissent au fil des âges à un rythme plus élevé pour les femmes que pour les hommes : alors que, dans la France contemporaine, après rupture d'union, les femmes refont leur vie cinq fois moins fréquemment si elles ont 50-54 ans plutôt que 25-29 ans, les hommes ne refont leur vie qu'une fois et demie moins fréquemment s'ils sont âgés de 50-54 ans plutôt que de 25-29 ans (Cassan, Mazuy et Clanché, 2001). Dans le même ordre d'idées, on a montré que "parmi les professionnels du spectacle [présents en couverture du magazine Paris-Match de 1949 à 2005], les caractéristiques d'âge sont très contrastées d'un sexe à l'autre. La tranche d'âge modale est celle des 45-49 ans chez les hommes, des 25-29 ans 
chez les femmes. Un tel écart suggère l'interprétation selon laquelle les qualités les plus valorisées chez les femmes sont le sex appeal et la beauté, dont il semble communément admis qu'ils se détériorent rapidement avec l'âge, tandis que chez les hommes le pouvoir de séduction résiste mieux à l'épreuve du vieillissement »(Chenu, 2008, p. 36).

Ce modèle de l'écart d'âge entre conjoints produit ainsi le résultat suivant (Bergstrom et Bagnoli, 1993). Chaque homme atteignant l'âge précoce décide de reporter sa mise en couple à l'âge tardif s'il anticipe entretemps un succès professionnel tel que le gain qu'il espère retirer du surcroît de désirabilité de la conjointe qu'il élit (gain lui-même permis par le surcroît de sa propre désirabilité aux yeux des conjointes potentielles) dépasse le coût qu'il s'attend à subir du fait qu'il renonce (d'ici là) aux gains d'une union. Â l'inverse, un homme atteignant l'âge précoce décide de se mettre en couple immédiatement s'il anticipe un succès professionnel qui n'est pas suffisamment éclatant pour compenser le temps d'attente avant la mise en couple. Du côté des hommes, donc, ceux qui s'attendent à bénéficier d'un succès professionnel d'ampleur inférieure à un certain niveau plancher se mettent en couple à l'âge précoce, et ceux qui s'attendent à un succès professionnel d'ampleur supérieure à ce niveau plancher se mettent en couple à l'âge tardif. Du côté des femmes, toutes se mettent en couple à l'âge précoce.

Si l'on considère une génération d'hommes, l'homme le plus désirable de sa génération se met en couple à l'âge tardif avec la femme d'âge précoce la plus désirable, l'homme qui est le deuxième plus désirable de sa génération se met en couple à l'âge tardif avec la femme d'âge précoce qui est la deuxième plus désirable, etc., jusqu'à ce qu'il n'y ait dans cette génération plus d'hommes qui s'attendaient à bénéficier d'un succès professionnel supérieur au niveau plancher - les hommes de cette génération qui s'attendaient à bénéficier d'un succès professionnel inférieur au niveau plancher se mettant pour leur part en couple dès l'âge précoce, et ce avec les femmes d'âge précoce qui n'étaient pas suffisamment désirables pour pouvoir attirer les hommes qui se mettaient alors en couple à l'âge tardif. Par conséquent, on observe un écart d'âge moyen entre conjoints au profit de l'homme qui est (entièrement) dû à l'écart d'âge entre conjoints au sein des couples dont les membres sont, respectivement, les plus désirables de leur génération.

Ce modèle explicatif, évidemment simplificateur à l'extrême, a pour mérite de pointer du doigt des mécanismes causaux susceptibles d'expliquer l'existence - le caractère universel - de l'écart d'âge moyen entre conjoints au profit de l'homme. Fondamentalement, selon ce modèle, ce serait l'écart entre les âges auxquels hommes et femmes connaissent leurs pics de désirabilité aux yeux des membres du sexe opposé qui serait à l'origine de l'écart d'âge entre conjoints. Comme le suggère l'article francophone de référence sur l'écart d'âge entre conjoints, «à l'âge des choix, une modeste différence de 3 ou 4 ans est suffisante pour lever l'incertitude sur le devenir socioprofessionnel d'un homme »(Bozon, 1990b, p. 573) ; et «tandis que, chez les (femmes) jeunes, l'homme mûr est valorisé parce que l'âge apparaît comme l'accoucheur de la position sociale de l'homme, inversement il y a dévaluation de la femme mûre 
par les hommes moins jeunes, parce que l'âge est ici le film sur lequel les traces d'une expérience conjugale antérieure sont irréversiblement fixées, et qu'il reste symboliquement borné par la fin de la vie fertile » (Bozon, 1990a, p. 350).

Mais ce modèle explicatif a aussi pour mérite de permettre d'expliquer les variations d'écart d'âge entre conjoints, entre sociétés et entre couples. Premièrement, comme nous l'avons vu, l'écart d'âge croît avec l'âge de l'homme à la mise en couple et décroît avec l'âge de la femme à la mise en couple. En effet, étant donné que, selon ce modèle, les hommes, quel que soit leur âge, préfèrent les femmes jeunes, il n'est pas étonnant que plus les hommes se mettent en couple à un âge tardif, plus leur choix se porte sur une femme relativement plus jeune qu'eux. C'est pourquoi «les hommes acceptent d'autant mieux de vivre avec une femme légèrement plus âgée qu'ils sont jeunes. Mais ils changent d'avis ensuite » (Ferrand, 2004, p. 93). De même, comme selon ce modèle les femmes préfèrent les hommes d'âge mûr, il n'est pas étonnant que plus les femmes se mettent en couple à un âge tardif, plus leur choix se porte sur un homme d'âge plus proche. C'est pourquoi, là encore, «cette préférence pour les hommes plus âgés, mise en avant par les jeunes femmes, s'atténue après la trentaine » (ibid.). Cette explication concorde aussi avec les déclarations des hommes et des femmes telles qu'analysées dans l'article susmentionné (Bozon, 1990a, 1990b).

Deuxièmement, l'écart d'âge croît avec la position sociale de l'homme, tout au moins dans les sociétés agraires. En effet, selon ce modèle les hommes n'ont intérêt à reporter leur mise en couple à un âge relativement tardif que s'ils anticipent entretemps un succès professionnel qui les valorisera suffisamment pour que cela «vaille le coût» d'attendre. Par conséquent, le fait d'anticiper une mobilité professionnelle ascendante devrait à la fois - si elle se réalise conduire les hommes à occuper une position sociale élevée et à se marier tardivement, ce report du mariage tendant (comme nous venons de le voir) à accroître l'écart d'âge entre conjoints. Dans cette perspective, on a montré qu'aux États-Unis, en 1980, les hommes qui sont mariés pour la première fois ont des salaires d'autant plus élevés qu'ils se sont mariés plus tard (ce qui n'est pas le cas pour les femmes), peut-être parce que les hommes qui anticipaient l'obtention des salaires les plus élevés ont retardé leur mariage en vue de se mettre en couple avec les femmes les plus désirables et ont finalement obtenu les salaires les plus élevés (Bergstrom et Schoeni, 1996). Selon ce modèle, le fait de ne pas anticiper de mobilité professionnelle ascendante devrait $a$ contrario conduire les hommes à connaître un plus faible écart d'âge entre conjoints à leur profit. De fait, en France, en 1999, les hommes de moins de trente ans détenus en prison ont, plus souvent que les autres hommes de leur âge, des conjointes plus âgées qu'eux (Cassan et Mary-Portas, 2002). Et, pour prendre un exemple extrême dans lequel sont inversées les différences hommes-femmes de critères de choix du conjoint, dans le village de l'île grecque de Karpathos, «ce qui pousse [dans le troisième quart du $\mathrm{XX}^{\mathrm{e}}$ siècle] certains hommes, et spécialement les pauvres, à épouser des filles dévalorisées par leur âge, c'est qu'ils peuvent profiter de la difficulté qu'elles ont à se marier pour réclamer davantage d'argent»(Vernier, 1977, p. 56). Cette explication 
des variations d'écart d'âge avec la position sociale semble aussi compatible avec l'observation selon laquelle les femmes se déclarent d'autant plus hostiles aux hommes plus jeunes qu'elles sont moins diplômées et au chômage (Bozon, 1990a, 1990b) : c'est que plus leur niveau de vie hors couple risque d'être réduit, plus il leur est important de s'assurer - par le biais d'une mise en couple avec un homme d'âge mûr - que leur conjoint leur procurera un certain confort et une certaine sécurité matérielle.

Troisièmement, l'écart d'âge est plus élevé dans les sociétés moins développées du point de vue socioéconomique. Selon ce modèle, l'une des raisons qu'ont les hommes de préférer les femmes jeunes est que ce sont les plus fertiles et celles dont la durée restante de fertilité est la plus longue - donc, les femmes qui ont la plus grande fécondité potentielle. De ce point de vue, il n'est pas étonnant que la baisse de la fécondité désirée qui accompagne le développement socioéconomique réduise l'importance pour les hommes de se mettre en couple avec une femme jeune, et réduise donc l'écart d'âge entre conjoints. Dans les pays du monde, lors des années 1990 et 2000, les variables les plus fortement corrélées avec l'écart d'âge moyen entre conjoints au profit de l'homme sont effectivement les variables d'intensité de la fécondité (indicateur conjoncturel de fécondité) et d'intensité d'utilisation de la contraception (taux de prévalence des méthodes de contraception) (Tableau I). Et au sein de 18 pays d'Afrique subsaharienne des années 1990, parmi les femmes en couple (mariées ou non), «l'appartenance à un couple très inégalitaire en âge [dont l'écart d'âge dépasse 15 ans au profit de l'homme] est associée négativement à la pratique contraceptive » (Barbieri et Hertrich, 2005, p. 751) ; selon le modèle proposé ici, cela pourrait s'expliquer par le fait que si l'homme a choisi une femme relativement plus jeune - donc à la plus grande fécondité potentielle -, ce n'est pas pour qu'elle utilise une contraception, mais bien pour qu'elle fasse plus d'enfants que n'en aurait faits une femme relativement moins jeune. Enfin, comme on l'a vu, l'écart d'âge tend à baisser depuis les années 1950 dans la plupart des pays du monde. Selon ce modèle, cela pourrait s'expliquer - comme la régularité précédente - par le fait que la baisse de la fécondité désirée au fil du temps a réduit l'importance pour les hommes de se mettre en couple avec une femme jeune, ou encore par le fait que la hausse de l'activité économique des femmes a pu réduire l'importance pour les femmes de se mettre en couple avec un homme d'âge mûr.

Au total, le modèle de Bergstrom et Bagnoli paraît relativement satisfaisant, puisqu'il permet d'expliquer de façon crédible non seulement l'existence d'un écart d'âge moyen entre conjoints au profit de l'homme, mais aussi les diverses variations d'écart d'âge que la littérature empirique a observées, aussi bien entre sociétés qu'au fil du temps ou entre couples d'une même société. Toutefois, la question reste ouverte de savoir si ce modèle explicatif est aussi performant pour ce qui concerne les variations d'écart d'âge entre les couples des sociétés industrielles ou tertiaires que pour ce qui concerne les variations d'écart d'âge entre les couples des sociétés agraires. En effet, on comprend bien pourquoi, dans les sociétés agraires, l'écart d'âge entre conjoints croît avec la position sociale de l'homme : c'est que, pour atteindre une position sociale élevée - typiquement, posséder des terres -, les hommes 
doivent passer du temps à travailler pour se constituer le capital nécessaire à l'achat d'une terre ; les hommes qui, dans ce contexte, ont atteint une position sociale élevée sont donc des hommes qui se sont mariés relativement tard et, comme nous le savons - en raison de leur préférence pour les femmes jeunes -, le report du mariage accroît chez les hommes l'écart d'âge avec leur conjointe. Mais, dans les sociétés industrielles et tertiaires, dans lesquelles les positions sociales élevées sont moins conditionnées par l'accumulation d'un capital physique au cours d'une période de travail, et plutôt conditionnées par l'accumulation d'un «capital humain » au cours d'une période d'études, il pourrait en aller autrement.

Les études ont ceci de spécifique, par rapport à une période de travail destinée à se constituer un capital, qu'elles se déroulent non seulement avec des membres du sexe opposé (plutôt qu'entre hommes voire entre femmes, comme ce peut être le cas du travail), mais aussi avec des membres de la même génération, ou tout au moins de la même classe d'âge. Ainsi, le fait de suivre des études surexpose les individus à des conjoints potentiels de même âge qu'eux. C'est pourquoi l'essor de l'importance et de la durée des études, en accroissant les opportunités de rencontre entre individus de mêmes âges, pourrait avoir contribué au fil du temps à réduire l'écart d'âge moyen entre conjoints dans la population globale, mais aussi à réduire l'écart d'âge tout particulièrement chez les individus qui poursuivent leurs études le plus longtemps (Blossfeld, 2009), réduisant voire inversant ainsi le gradient social de l'écart d'âge entre conjoints. Comme on le sait, «n'importe qui ne "choisit" pas n'importe quel lieu pour "choisir" son conjoint» (Bozon et Héran, 1988, p. 121), et les membres des couches sociales supérieures tendent à privilégier des lieux dans lesquels on n'entre que par sélection (ou cooptation), au premier chef leur lieu d'études.

Plus précisément, il existe au moins deux raisons pour lesquelles l'essor de la durée des études aurait pu modifier le gradient social de l'écart d'âge entre conjoints. D'une part, comme nous venons de le voir, l'allongement de la scolarité dans un cadre mixte pourrait accroître la probabilité pour les étudiants - et particulièrement ceux qui poursuivent les études les plus longues - de rencontrer un conjoint d'âge proche sinon identique ; c'est ce que l'on peut appeler un « effet institution » des études. D'autre part, l'allongement de la scolarité féminine, en accroissant les revenus des femmes (en eux-mêmes, et par rapport à ceux des hommes), pourrait réduire aux yeux des femmes - et particulièrement aux yeux des femmes les plus diplômées, donc les mieux rémunérées - l'attractivité des hommes d'âge mûr, qui sont plus sécurisants matériellement; c'est ce que l'on peut appeler un « effet capital humain »des études pour les femmes (17).

(17) Notons que si chacun de ces deux effets contredit la prédiction statique du modèle Bergstrom-Bagnoli selon laquelle l'écart d'âge entre conjoints devrait croître avec la position sociale du couple, leurs statuts diffèrent : alors que 1 '«effet institution » des études est fonda- mentalement extérieur à ce modèle, l' « effet capital humain » des études des femmes n'est en fait que la prédiction dynamique du modèle Bergstrom- Bagnoli sous l'hypothèse qu'au fil du temps le niveau d'études des hommes détermine de moins en moins leur désirabilité. 
Alors que, dans les sociétés agraires, l'écart d'âge entre conjoints croît avec la position sociale de l'homme, dans les sociétés industrielles et tertiaires, il pourrait donc décroître avec le niveau d'études - donc avec la position sociale - des conjoints (Van Poppel et al., 2001). De fait, certaines analyses suggèrent que, dans la France contemporaine, l'écart d'âge entre conjoints pourrait décroître avec le niveau d'études. Dans les premières unions en 1984, les femmes étaient moins diplômées dans les couples dans lesquels l'écart d'âge entre conjoints dépasse trois ans que dans les couples dans lesquels l'écart d'âge est négatif (Bozon, 1991). Et, sur plus longue période, chez les couples formés des années 1930 aux années 1990, l'écart d'âge entre conjoints semble décroître à mesure que croît le niveau relatif d'études (soit, le niveau d'études des individus par rapport au niveau d'études moyen des membres de leur génération) (Vanderschelden, 2006). Si de telles observations se confirmaient et s'expliquaient par l' «effet institution » des études, le modèle Bergstrom-Bagnoli pourrait donc se révéler moins performant pour expliquer les variations d'écart d'âge entre couples des sociétés contemporaines que pour expliquer celles des sociétés agraires.

\section{L'écart d'âge entre conjoints en France en 1978-1998 : un test empirique du modèle explicatif}

Dans quelle mesure le modèle Bergstrom-Bagnoli - qui semble relativement satisfaisant pour comprendre les variations d'écart d'âge entre conjoints au sein des sociétés agraires - permet-il aussi de comprendre ces variations dans la France contemporaine ? L'allongement de la durée d'études dans les sociétés industrielles et tertiaires n'aurait-il pas - par les biais d'un « effet institution » des études et d'un « effet capital humain» des études pour les femmes - inversé le gradient social de l'écart d'âge entre conjoints ? Pour le savoir, nous exploitons les données fournies par l'enquête Étude de l'histoire familiale 1999 (Insee).

\section{Statistiques descriptives de l'écart d'âge entre conjoints}

Parmi les individus enquêtés, nous ne sélectionnons pour nos analyses que les individus des générations 1935 à 1968 qui se sont mis en couple (que ce soit pour la première fois ou non, et que ce soit par un mariage direct ou par une cohabitation) dans les vingt années précédant l'enquête, de 1978 à 1998. En effet, comme l'enquête ne précise l'écart d'âge entre l'enquêté et son conjoint que pour une seule des unions de l'enquêté (la première s'il n'en a connu qu'une seule, la dernière s'il en a connu plus d'une), les informations dont on dispose concernant les unions formées il y a longtemps tendent à surreprésenter les unions les plus longues ; il convient donc de se concentrer sur les unions formées relativement récemment, pour lesquelles on dispose d'informations moins fortement affectées par ce biais de sélection. On exclut 
par ailleurs de nos analyses les immigrés, dont les comportements de mise en couple pourraient obéir à des logiques spécifiques qui ne sont pas notre objet.

$\mathrm{Au}$ total, notre échantillon comprend 41059 hommes et 39591 femmes nés en moyenne autour de la fin des années 1950 et mis en couple autour du milieu des années 1980, et dont les caractéristiques sont présentées dans le Tableau II. Dans cet échantillon, les hommes se sont mis en couple en moyenne à 28,3 ans et les femmes à 26,6 ans, les trois quarts environ de ces mises en couple constituant la première des enquêtés (si bien que plus des trois quarts des enquêtés n'ont jamais eu d'enfant avant cette mise en couple). Plus des deux tiers de ces mises en couple ont débuté par une cohabitation plutôt que par un mariage direct, et lors de leur mise en couple plus de $90 \%$ de ces individus avaient déjà exercé une activité professionnelle.

Parmi toutes ces mises en couple, celles des enquêtés hommes comprennent un écart d'âge moyen entre conjoints de 2,6 ans, et celles des enquêtées femmes un écart d'âge moyen de 1,9 an. Cette différence s'explique largement par le fait que, même si l'écart d'âge moyen entre conjoints au sein des premières unions est proche quel que soit le sexe des enquêtés $(2,05$ ans pour les hommes, 2,24 ans pour les femmes), l'écart d'âge entre conjoints au sein des unions ultérieures est sensiblement différent selon que l'enquêté est un homme $(4,69$ ans) ou une femme (1,06 an) : c'est là l'une des conséquences du fait que, comme nous l'avons vu, l'âge à la mise en couple accrôit l'écart d'âge entre conjoints chez les hommes, et le réduit chez les femmes. 
TABLEAU II. - Statistiques descriptives des individus non immigrés des générations 1935 à 1968 mis en couple au moins une fois à partir de 15 ans de 1978 à 1998 dans l'enquête Étude de l'histoire familiale : distribution des modalités des variables qualitatives, et moyenne (et écart type) des variables quantitatives

\begin{tabular}{|c|c|c|c|}
\hline & & $\begin{array}{c}\text { HOMMES } \\
(\mathrm{N}=41059)\end{array}$ & $\begin{array}{c}\text { FEMMES } \\
(\mathrm{N}=39591)\end{array}$ \\
\hline Génération & & $1958,7(6,8)$ & $1959,7(6,4)$ \\
\hline \multirow{11}{*}{$\begin{array}{l}\text { Niveau de diplôme lors } \\
\text { de la mise en couple }\end{array}$} & Aucun diplôme & $11,4 \%$ & $10,8 \%$ \\
\hline & CEP & $5,3 \%$ & $5,8 \%$ \\
\hline & BEPC & $8,1 \%$ & $9,8 \%$ \\
\hline & CAP & $24,7 \%$ & $13,9 \%$ \\
\hline & BEP & $10,8 \%$ & $11,9 \%$ \\
\hline & Bac général & $4,1 \%$ & $6,5 \%$ \\
\hline & Bac techno. ou pro. & $7,4 \%$ & $7,5 \%$ \\
\hline & Dipl. univ. de $1^{\text {er }}$ cycle & $8,9 \%$ & $11,7 \%$ \\
\hline & Dipl. univ. de $2^{\mathrm{e}}$ ou $3^{\mathrm{e}}$ cycles & $10,0 \%$ & $7,1 \%$ \\
\hline & Étudiant & $5,4 \%$ & $11,1 \%$ \\
\hline & NR & $3,8 \%$ & $3,7 \%$ \\
\hline \multirow{2}{*}{$\begin{array}{l}\text { Statut d'activité lors } \\
\text { de la mise en couple }\end{array}$} & N'a jamais été actif & $3,2 \%$ & $9,5 \%$ \\
\hline & A déjà été actif & $96,8 \%$ & $90,5 \%$ \\
\hline \multirow{7}{*}{ PCS } & Agriculteur & $3,5 \%$ & $1,5 \%$ \\
\hline & Indépendant & $8,1 \%$ & $3,8 \%$ \\
\hline & Cadre ou PIS & $17,8 \%$ & $9,7 \%$ \\
\hline & Profession intermédiaire & $24,1 \%$ & $24,1 \%$ \\
\hline & Employé & $12,4 \%$ & $48,4 \%$ \\
\hline & Ouvrier & $33,9 \%$ & $10,2 \%$ \\
\hline & Inactif & $0,1 \%$ & $2,3 \%$ \\
\hline Année de la mise en couple & & $1987(5,6)$ & $1986,3(5,5)$ \\
\hline Âge à la mise en couple & & $28,3(7,2)$ & $26,6(7,3)$ \\
\hline \multirow[b]{2}{*}{ Rang de l'union } & Première union & $77,3 \%$ & $74,6 \%$ \\
\hline & Union ultérieure & $22,7 \%$ & $25,4 \%$ \\
\hline \multirow{2}{*}{$\begin{array}{l}\text { A déjà eu des enfants } \\
\text { avant la mise en couple }\end{array}$} & Non & $82 \%$ & $78,2 \%$ \\
\hline & Oui & $18 \%$ & $21,8 \%$ \\
\hline \multirow[b]{2}{*}{ Modalité de mise en couple } & Cohabitation & $71,6 \%$ & $69,6 \%$ \\
\hline & Mariage direct & $28,4 \%$ & $30,4 \%$ \\
\hline $\begin{array}{l}\text { Écart d'âge entre conjoints } \\
\text { au profit de l'homme }\end{array}$ & & $2,6(5,2)$ & $1,9(5,5)$ \\
\hline
\end{tabular}


Notons que, sans surprise, on retrouve dans cet échantillon les mêmes variations d'écart d'âge entre conjoints selon les âges des conjoints à la mise en couple que nous avions observées, dans la Figure II, à partir des données d'état-civil portant sur l'année 2007 : l'écart d'âge entre conjoints au profit de l'homme croît avec l'âge de l'homme à la mise en couple et devient positif dès que les hommes se mettent en couple à partir d'une vingtaine d'années, tandis que l'écart d'âge entre conjoints au profit de la femme se réduit progressivement à mesure qu'augmente l'âge de la femme à la mise en couple et ne devient positif - et encore, de très peu - qu'à partir du moment où les femmes dépassent la quarantaine d'années lors de leur mise en couple (Figure V).

\section{FIGURE V. - Écart d'âge moyen entre conjoints selon l'âge des conjoints à la mise en couple - France, 1978-1998}

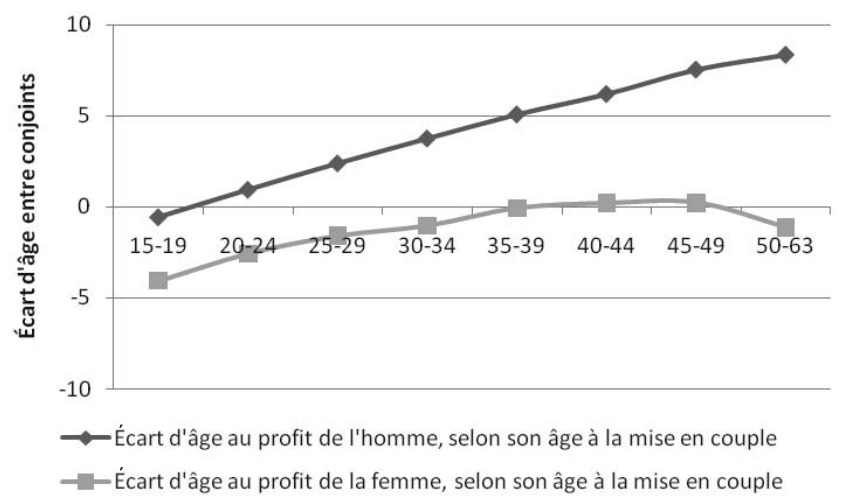

Champ : Individus non immigrés des générations 1935 à 1968 mis en couple au moins une fois à partir de 15 ans de 1978 à 1998.

Source : Enquête Étude de l'histoire familiale 1999 (Insee).

En revanche, à partir de simples statistiques descriptives, on n'observe pas le phénomène selon lequel l'écart d'âge entre conjoints croîtrait avec la position sociale du couple, que celle-ci soit mesurée par le niveau de diplôme ou la catégorie socioprofessionnelle des conjoints. Notamment, l'écart d'âge entre conjoints ne varie que peu au fil des niveaux de diplôme masculins, aucune tendance notable - ni croissante, ni décroissante - ne semblant se dégager.

On observe toutefois un autre phénomène, selon lequel les individus qui étaient encore étudiants lors de leur mise en couple diffèrent sensiblement des individus qui avaient achevé leurs études lors de leur mise en couple (que ce soit avec ou sans diplôme). En effet, parmi les premières unions masculines, 
l'écart d'âge moyen entre conjoints est sensiblement plus faible lorsque l'homme est encore étudiant lors de sa mise en couple $(0,44$ an) que lorsqu'il a terminé ses études (d'un minimum de 1,82 an lorsqu'il a obtenu un bac technologique ou professionnel à un maximum de 3,16 ans lorsqu'il n'a obtenu que le CEP). Cette observation est compatible avec l'idée selon laquelle le lieu d'études constituant un lieu de recrutement du conjoint caractérisé par sa forte ségrégation par âges, il favorise des mises en couple avec des écarts d'âge faibles sinon nuls. Une telle interprétation tend à être confirmée par le fait que, parmi les premières unions masculines comme féminines, la dispersion de l'écart d'âge entre conjoints est plus faible lorsque les individus sont encore étudiants lors de leur mise en couple, plutôt que lorsqu'ils ont terminé leurs études : alors que l'écart type de l'écart d'âge entre conjoints (soit, la variation moyenne autour de la moyenne de l'écart d'âge) est, tous niveaux de diplôme à la mise en couple confondus, de 4,4 ans pour les premières unions masculines et de 4,7 ans pour les premières unions féminines, il n'est respectivement que de 3 et de 3,8 ans pour les premières unions débutées alors que les enquêtés étaient encore étudiants. Bref, les étudiants forment des couples dans lesquels l'écart d'âge au profit de l'homme est relativement faible et relativement peu variable, conformément à un possible «effet institution » de la scolarité sur l'écart d'âge entre conjoints (18).

Cela dit, parmi les premières unions féminines, l'écart d'âge moyen entre conjoints est plus élevé lorsque la femme est encore étudiante lors de sa mise en couple (2,9 ans) plutôt que lorsqu'elle a terminé ses études (d'un minimum de 1,83 an lorsqu'elle a obtenu le CEP à 2,52 ans lorsqu'elle n'a obtenu aucun diplôme). Si cette observation peut sembler étonnante, elle pourrait en partie être due au fait que les étudiantes qui se mettent en couple sont, le plus souvent, relativement jeunes - or, on le sait, les femmes qui se mettent en couple jeunes choisissent de préférence des hommes sensiblement plus âgés qu'elles. Pour permettre de départager les influences de ces diverses caractéristiques individuelles sur l'écart d'âge entre conjoints, et plus généralement pour affiner nos observations, il devient ici nécessaire de procéder à des analyses statistiques plus sophistiquées.

\section{Analyse de variance de l'écart d'âge entre conjoints}

Pour cela, nous estimons, séparément pour les hommes (Tableau III) et pour les femmes (Tableau IV), des modèles emboîtés d'analyse de variance dans lesquels la variable à expliquer est l'écart d'âge entre conjoints au profit

(18) De même que les individus plus qualifiés sont plus homogames du point de vue de leurs âges, ils sont, comme le montre une revue de la littérature empirique sur le sujet, plus homogames du point de vue de leurs niveaux d'études (Blossfeld, 2009). Dans les deux cas, le même «effet institution » des études est en cause : faire des études plus longues expose plus longtemps - et aux âges plus pertinents - à des rencontres avec des conjoints de même âge et de même niveau de diplôme. 
de l'homme, calculé comme la différence entre l'année de naissance de la femme et celle de l'homme du couple.

Les variables explicatives que nous introduisons successivement dans ces modèles statistiques sont destinées à tester les prédictions empiriques du modèle Bergstrom-Bagnoli (19), ainsi que le possible « effet institution » des études. Selon ce modèle, le niveau de diplôme des hommes, en accroissant leur âge à la mise en couple, devrait accroître l'écart d'âge entre conjoints ; toutefois, cet effet positif du niveau d'études sur l'écart d'âge devrait s'annuler - ou tout au moins se réduire - une fois que l'on a contrôlé l'âge de l'homme à la mise en couple. Selon l' «effet capital humain » des études des femmes, cohérent avec le modèle, le fait d'avoir obtenu un diplôme plus élevé, en accroissant le niveau de vie et donc en réduisant l'attractivité des hommes d'âge mûr, devrait réduire l'écart d'âge, et cet effet négatif devrait en partie au moins subsister lorsque l'on contrôle l'âge de la femme à la mise en couple. À l'inverse, selon l' "effet institution» des études, extérieur au modèle Bergstrom-Bagnoli, le fait d'être encore étudiant lors de la mise en couple ou d'avoir poursuivi ses études plus longtemps, en accroissant la probabilité d'avoir rencontré un conjoint d'âge proche, devrait réduire l'écart d'âge, et cet effet négatif devrait en partie au moins subsister lorsque l'on contrôle l'âge de l'homme ou de la femme à la mise en couple.

Dans la même ligne d'idées, selon le modèle, le fait pour un homme de ne pas encore avoir débuté sa carrière professionnelle (plutôt que d'être déjà entré sur le marché du travail), en le rendant moins sécurisant matériellement pour ses conjointes potentielles, devrait le conduire à se mettre en couple avec des femmes relativement moins jeunes par rapport à lui, donc devrait réduire l'écart d'âge entre conjoints ; toutefois, comme le fait de ne pas encore avoir débuté sa carrière professionnelle est le fait d'hommes relativement jeunes, cet effet négatif devrait se réduire une fois que l'on a contrôlé l'âge de l'homme à la mise en couple. Parallèlement, le fait pour une femme de ne pas encore avoir débuté sa carrière professionnelle, en la rendant plus dépendante des revenus de son conjoint, devrait accroître l'écart d'âge entre conjoints ; et, là encore, comme le fait de ne pas encore avoir débuté sa carrière professionnelle concerne des femmes relativement jeunes, cet effet positif devrait se réduire une fois que l'on a contrôlé l'âge de la femme à la mise en couple.

(19) Nous ne testons ici que les prédictions de ce modèle, et non pas ses hypothèses, dont nous avons vu ci-dessus qu'elles étaient largement validées par la littérature empirique internationale sur les préférences des hommes et des femmes en matière de choix du conjoint. 
Jean-François MIGNOT

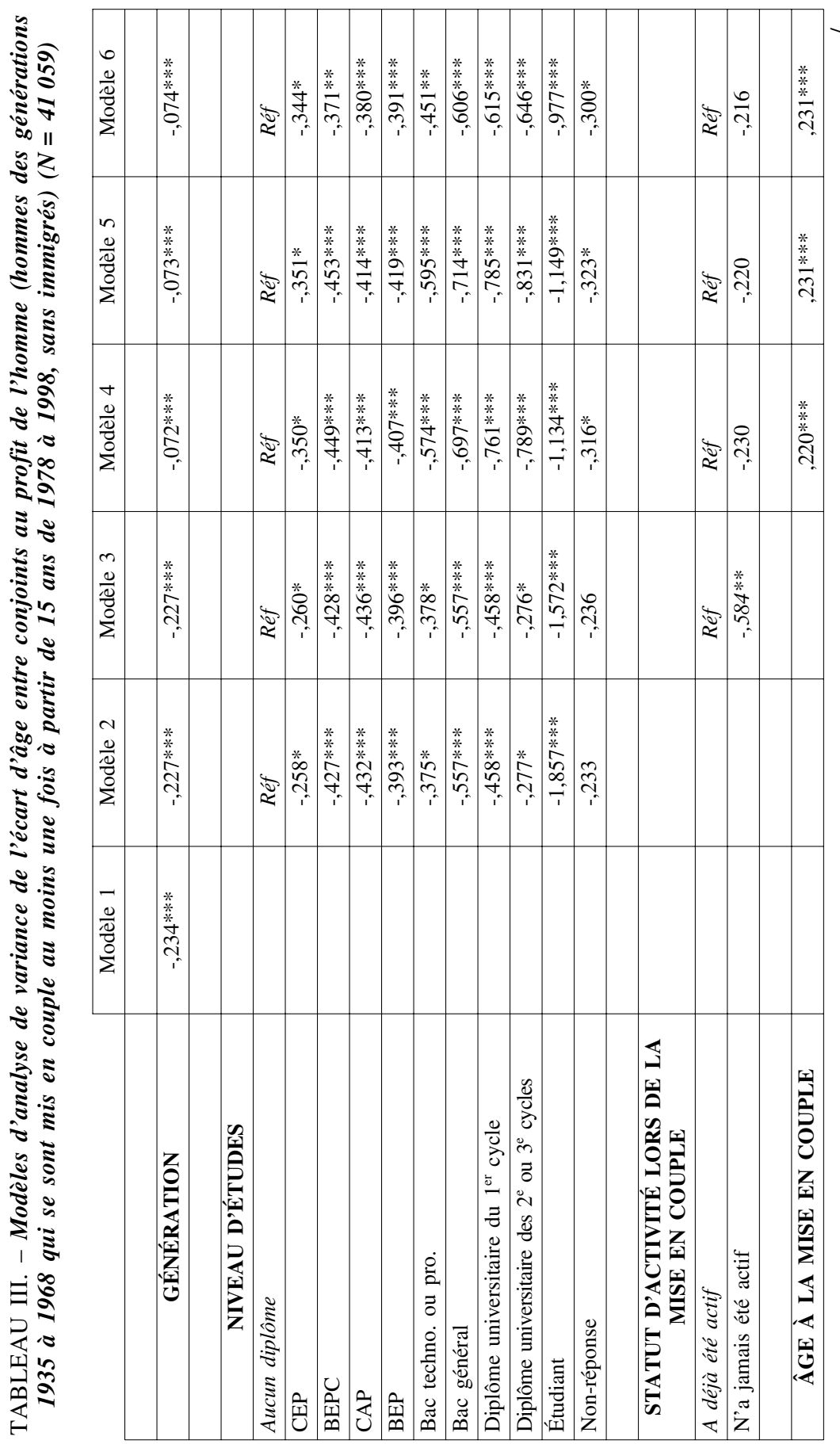


Revue française de sociologie

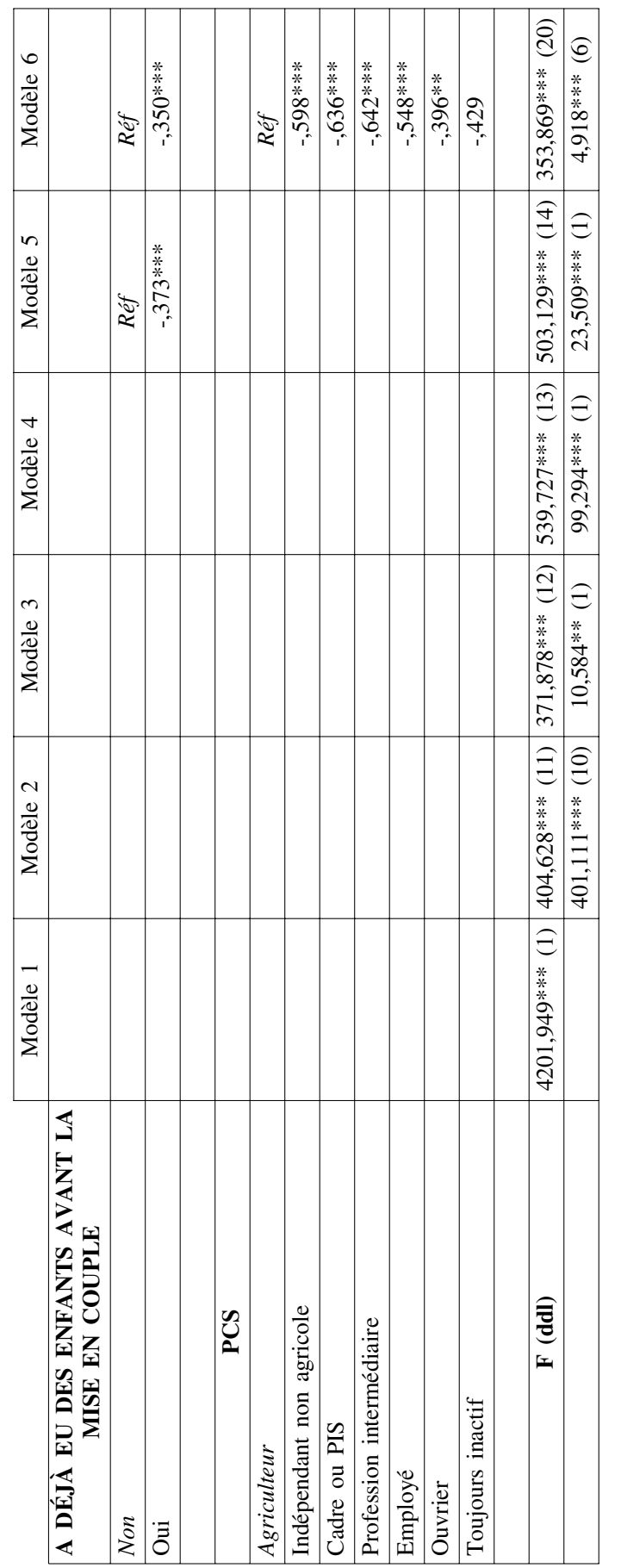




Jean-François MIGNOT

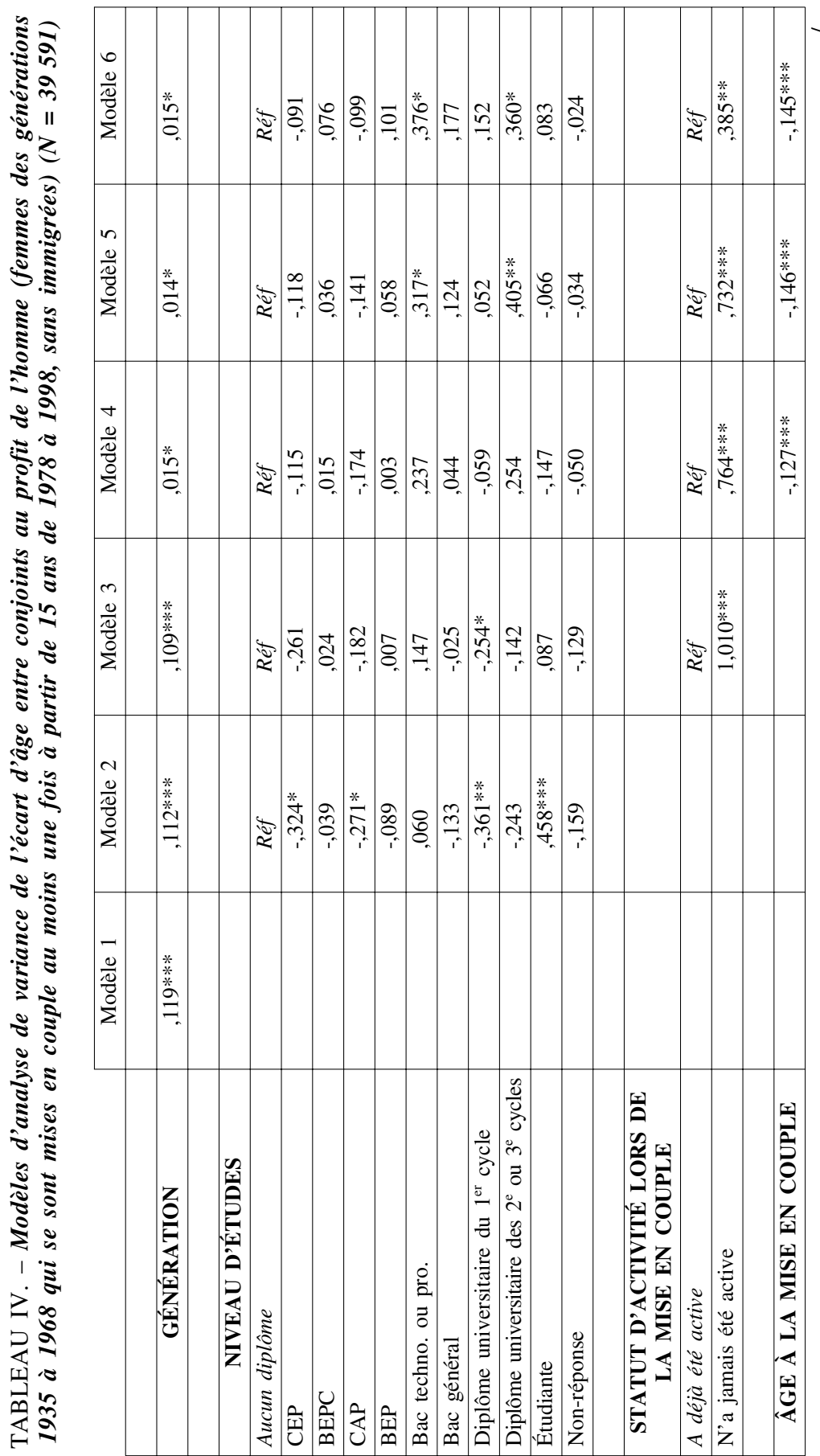


Revue française de sociologie

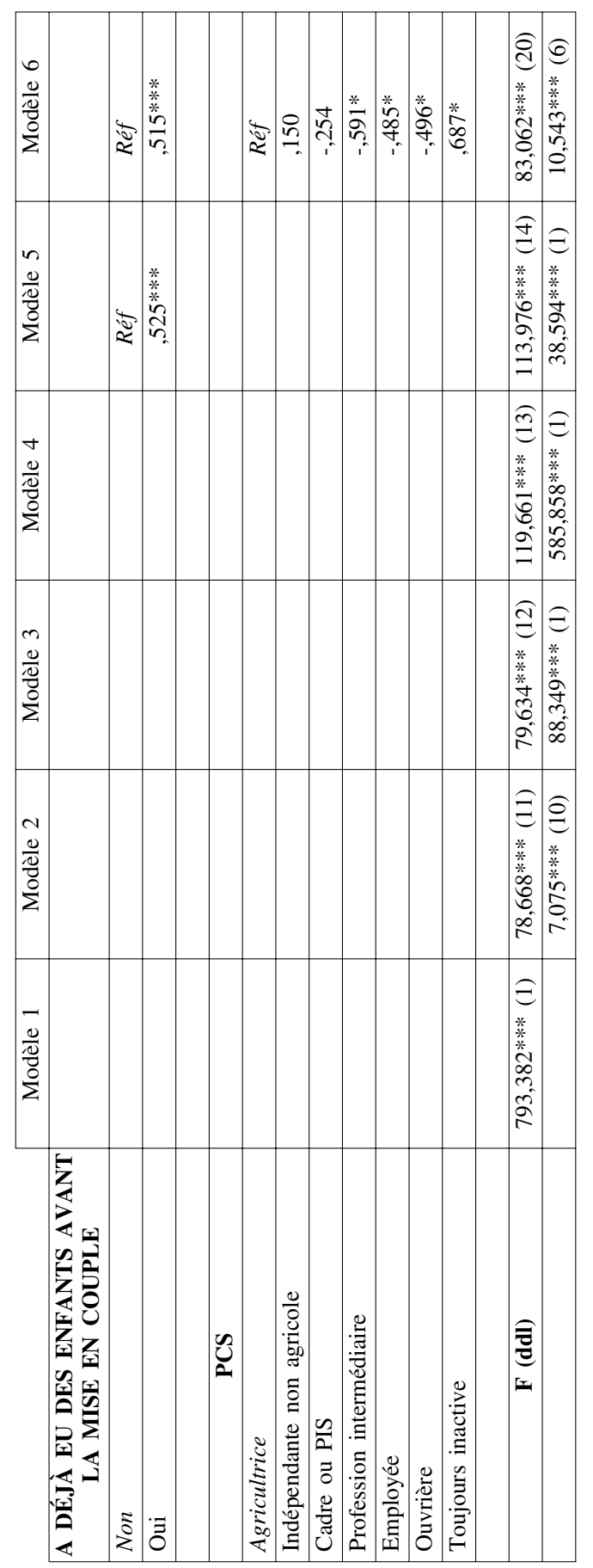

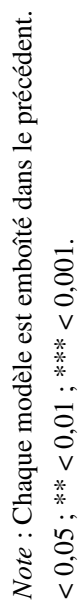


À partir d'un modèle initial de l'écart d'âge entre conjoints ne comprenant comme variable explicative que la génération des enquêtés (modèle 1), nous introduisons la variable de niveau d'études (20). Chez les hommes (modèle 2 du Tableau III), le fait d'être encore étudiant lors de la mise en couple (plutôt que d'avoir terminé ses études) et le fait d'avoir obtenu un diplôme à l'issue des études (plutôt que de n'avoir obtenu aucun diplôme) réduisent de façon sensible l'écart d'âge entre conjoints, ce qui est incompatible avec le modèle Bergstrom-Bagnoli, mais conforme à un « effet institution» des études. Chez les femmes (modèle 2 du Tableau IV), le fait d'être encore étudiante lors de la mise en couple accroît l'écart d'âge entre conjoints - ce qui semble contraire à un « effet institution » des études -, et même si les effets de plusieurs modalités de diplôme ne sont pas significatifs, le fait d'avoir obtenu certains diplômes à l'issue des études tend lui aussi à réduire l'écart d'âge entre conjoints, ce qui semble conforme à un «effet capital humain » des études des femmes.

À même niveau de diplôme, le fait d'être encore inactif (plutôt que d'être déjà entré sur le marché du travail) a les effets prévus sur l'écart d'âge entre conjoints. Chez les hommes (modèle 3 du Tableau III), le fait d'être encore inactif réduit l'écart d'âge entre conjoints, conformément à l'idée selon laquelle des hommes qui n'ont pas encore entamé leur carrière ne pourraient attirer que des femmes suffisamment âgées pour ne pas avoir à dépendre d'eux matériellement. Chez les femmes (modèle 3 du Tableau IV), le fait d'être encore inactive accroît l'écart d'âge entre conjoints, conformément à la même idée selon laquelle des femmes dépendantes matériellement de leur conjoint tendent à choisir des hommes suffisamment âgés pour être sécurisants.

Mais qu'advient-il de ces relations statistiques lorsque l'on contrôle l'âge à la mise en couple ? Notons tout d'abord que, comme prévu par le modèle Bergstrom-Bagnoli (modèle 4), l'âge des hommes à la mise en couple accrôit très fortement l'écart d'âge entre conjoints (et de façon très fortement significative), tandis que l'âge des femmes à la mise en couple le réduit fortement

(20) Même si les coefficients associés à la variable de génération des enquêtés ne présentent pas ici d'intérêt majeur - cette variable n'est introduite que dans le but que l'effet des autres variables soit mesuré au sein de chaque génération -, il convient d'expliquer pourquoi les hommes semblent connaître un écart d'âge entre conjoints de plus en plus réduit au fil des générations - ce qui est conforme aux observations sur données agrégées - alors que les femmes semblent connaître un écart d'âge entre conjoints de plus en plus élevé au fil des générations - ce qui ne l'est pas. La raison tient à la composition de notre échantillon. En effet, plus les hommes sont d'une génération récente, moins ils ont eu l'occasion de se remettre en couple à des âges élevés (or, dans le cas où un enquêté s'est remis en couple, c'est bien cette dernière mise en couple que nous observons, et non pas sa première mise en couple); comme l'âge des hommes à la mise en couple accroît l'écart d'âge, plus les hommes sont d'une génération récente, moins ils ont eu de chances de connaître des écarts d'âge élevés. À l'inverse, comme l'âge des femmes à la mise en couple réduit l'écart d'âge, plus les femmes sont d'une génération récente, moins elles ont eu l'occasion de connaître des écarts d'âge réduits. C'est ce dont témoigne le fait que, pour les hommes comme pour les femmes, l'introduction de la variable d'âge à la mise en couple (modèles 4) réduit très sensiblement l'ampleur du coefficient associé à la variable génération. 
(et de façon particulièrement significative). Plus précisément, chez les hommes chaque année d'âge supplémentaire à la mise en couple tend à accroître l'écart d'âge avec leur conjointe de 0,22 an, tandis que chez les femmes chaque année d'âge supplémentaire à la mise en couple tend à réduire l'écart d'âge avec leur conjoint de 0,12 an (modèles 4). Conformément au modèle, l'âge à la mise en couple reste, de loin, le déterminant majeur de l'écart d'âge, ce qui s'explique vraisemblablement par le fait que les hommes préfèrent les femmes jeunes - donc plus ils se mettent en couple tard, plus les femmes qu'ils estiment attractives sont jeunes par rapport à eux - et par le fait que les femmes préfèrent les hommes mûrs - donc plus elles se mettent en couple tard, moins les hommes qu'elles estiment sécurisants sont âgés par rapport à elles. Le modèle Bergstrom-Bagnoli apparaît ici satisfaisant, puisqu'il propose la seule explication disponible aux effets - robustes et prononcés - de l'âge à la mise en couple sur l'écart d'âge entre conjoints.

Chez les hommes (modèle 4 du Tableau III), à âge donné de mise en couple, le fait d'être encore étudiant à la mise en couple et le niveau de diplôme obtenu à l'issue des études tendent à réduire l'écart d'âge entre conjoints. Il semble donc clair que, pour ce qui concerne les hommes, le gradient social de l'écart d'âge entre conjoints s'est inversé dans la France contemporaine par rapport à ce qu'il était jusque vers la première moitié du $\mathrm{XX}^{\mathrm{e}}$ siècle. Alors que, dans les sociétés agraires, les hommes les plus dotés en capitaux socioéconomiques étaient ceux dont la conjointe était relativement la plus jeune - parce que, pour acquérir ces capitaux, ils devaient reporter leur mise en couple, ce qui les conduisait à élire une conjointe relativement jeune -, désormais les hommes les plus dotés en capitaux scolaires (ainsi que les étudiants) sont ceux dont la conjointe est relativement la moins jeune, parce que leur lieu d'études favorise le recrutement de conjointes d'âge proche du leur (21). Ces observations, incompatibles avec le modèle Bergstrom-Bagnoli, s'expliquent bien par un «effet institution » des études. Apparaît ainsi une faiblesse du modèle : même s'il explique bien la direction du gradient social de l'écart d'âge entre conjoints dans les sociétés agraires, seul l' « effet institution » des études permet d'expliquer le retournement - et la nouvelle direction du gradient social de l'écart d'âge entre conjoints dans la France contemporaine.

La seule analyse poussée qui a été menée sur cet «effet institution » des études tend à conforter l'interprétation proposée ici. En effet, sur données brutes, il apparaît qu'aux Pays-Bas, dans la seconde moitié du $\mathrm{XX}^{\mathrm{e}}$ siècle, les conjoints très qualifiés ont plus souvent fréquenté les mêmes institutions d'enseignement que les conjoints moins qualifiés, et que les conjoints ayant fréquenté les mêmes institutions d'enseignement - qui constituent une part croissante des couples - connaissent un écart d'âge relativement faible

(21) Par ailleurs, une fois que l'on contrôle par l'âge à la mise en couple, le fait pour les hommes de n'être pas encore entrés sur le marché du travail ne réduit plus significativement l'écart d'âge entre conjoints, peut-être parce que les hommes qui se mettent en couple avant leur entrée sur le marché du travail sont trop peu nombreux pour qu'un tel effet soit ici mesurable (d'autant que la modalité « étudiant» est aussi incluse dans le modèle). 
(Smeenk, 1998). En outre, des analyses multivariées indiquent que, à même niveau de diplôme, le fait d'avoir fréquenté la même institution d'enseignement supérieur accroît la probabilité que les conjoints soient de mêmes âges ou d'âges très proches (ce qui n'est pas le cas des conjoints ayant fréquenté les mêmes institutions d'enseignement primaire ou secondaire, ou ayant résidé dans le même quartier) (ibid.). Le lieu d'études semble ainsi constituer un « marché matrimonial local » (ibid.) d'importance tout particulière pour les plus diplômés, et d'importance croissante au fil des promotions de mise en couple.

Chez les femmes (modèle 4 du Tableau IV), à âge donné de mise en couple, ni le fait d'être encore étudiante, ni le niveau de diplôme obtenu à l'issue des études n'ont d'effet significatif sur l'écart d'âge, ce qui implique que, pour elles, ni l' «effet institution», ni l' «effet capital humain » des études ne sont véritablement observés. Apparemment, le fait pour une femme d'être étudiante (plutôt que d'avoir terminé ses études) ne modifie pas sensiblement ses critères de choix du conjoint en matière d'âge, et le fait d'avoir obtenu un diplôme relativement élevé (plutôt qu'un diplôme de niveau inférieur) ne réduit pas sensiblement sa préférence pour des hommes d'âge mûr (ce serait même plutôt le contraire pour les diplômes universitaires les plus élevés, dans les modèles 5 et 6 ). De ce point de vue, le fait que le modèle Bergstom-Bagnoli néglige les variations de niveau d'études des femmes n'apparaît pas dommageable : de fait, elles n'affectent pas sensiblement l'écart d'âge entre conjoints. En outre, le fait de contrôler par l'âge à la mise en couple réduit mais n'annule pas l'effet positif du fait de ne pas encore avoir débuté sa carrière professionnelle, ce qui tend à confirmer que pour une femme le fait de ne pas encore avoir débuté sa carrière professionnelle, en la rendant plus dépendante des revenus de son conjoint, l'incite à choisir un conjoint d'âge suffisamment mûr pour être sécurisant matériellement. Il s'agit là d'une observation qui prolonge directement le modèle Bergstom-Bagnoli, puisque selon ce dernier la préférence des femmes pour des hommes plus âgés proviendrait du souci d'accéder à un niveau de vie satisfaisant.

Plusieurs analyses non reportées ici confirmant le résultat déjà obtenu selon lequel c'est moins le rang de l'union (première union ou union ultérieure) que le fait d'avoir déjà eu des enfants qui modifie l'écart d'âge entre conjoints dans la France contemporaine (Vanderschelden, 2006), nous introduisons une variable indiquant si l'enquêté a - ou non - déjà eu des enfants à la date de sa mise en couple (modèle 5). À ce propos, en admettant - avec le modèle Bergstrom-Bagnoli - que l'âge des hommes est en partie perçu par les femmes comme un indicateur de position sociale et que l'âge des femmes est en partie perçu par les hommes comme un indicateur de fécondité potentielle, on peut s'attendre à observer deux phénomènes. D'une part, le fait pour un homme d'avoir déjà eu des enfants dans un couple précédent, en réduisant son désir d'avoir des enfants dans son nouveau couple, devrait réduire le degré auquel il préfère une conjointe plus jeune que lui, ce qui devrait réduire l'écart d'âge. D'autre part, le fait pour une femme d'avoir la charge des enfants qu'elle a eus dans un couple précédent, en accroissant la valeur qu'elle accorde à la sécurité matérielle que peut lui procurer un conjoint, 
devrait accroître le degré auquel elle préfère un conjoint plus âgé qu'elle, ce qui devrait accroître l'écart d'âge.

Effectivement, à même génération, même âge à la mise en couple, même niveau d'études et même statut d'activité lors de la mise en couple, le fait pour un homme d'avoir déjà eu des enfants tend à réduire (de façon très fortement significative) l'écart d'âge entre conjoints (modèle 5 du Tableau III), tandis que pour une femme le fait d'avoir déjà eu des enfants tend à accroître (de façon très fortement significative) l'écart d'âge entre conjoints (modèle 5 du Tableau IV). En outre, l'introduction de cette variable dans les modèles ne modifie que peu les coefficients associés aux variables de niveau d'études, de statut d'activité et d'âge à la mise en couple, ce qui signifie que les régularités que nous avons observées entre ces variables et l'écart d'âge sont relativement robustes. Ces observations tendent à confirmer que, parmi les motivations sous-jacentes à l'existence et aux variations de l'écart d'âge entre conjoints, se trouvent la préférence des hommes pour des femmes plus fertiles et encore fertiles plus longtemps, et la préférence des femmes pour des hommes mûrs donc plus sécurisants matériellement.

Enfin, dans un dernier modèle, nous examinons si le gradient social de l'écart d'âge entre conjoints est effectivement inverse à celui qui prévaut dans les sociétés agraires (et qui est prévu par le modèle Bergstrom-Bagnoli), mais en utilisant non plus le seul niveau de diplôme, mais aussi la catégorie socioprofessionnelle des individus. Notons que nous n'avons pas utilisé cet indicateur dans les premiers modèles car la catégorie que renseigne l'enquête est la catégorie socioprofessionnelle à la date de l'enquête, et non pas à la date de la mise en couple, ce qui expose cette variable à toute une série de biais. Quoi qu'il en soit, à même niveau de diplôme et à autres variables contrôlées, pour les hommes c'est chez les agriculteurs, et pour les femmes chez les inactives (puis chez les agricultrices, voire les indépendantes non agricoles) que l'écart d'âge entre conjoints est le plus élevé (modèles 6). Cela pourrait en partie s'expliquer par le fait qu'il s'agirait là des individus qui ont la plus grande fécondité désirée, ce qui à la fois inciterait ces hommes à choisir des femmes relativement jeunes (donc plus fertiles, et encore fertiles pendant plus longtemps) et inciterait ces femmes à choisir des hommes relativement mûrs (donc mieux à même d'assumer la charge de plusieurs enfants). En effet, dans notre échantillon, les hommes les plus féconds sont bien les agriculteurs (1,9 enfant en moyenne, contre 1,81 dans la population générale), et les femmes les plus fécondes sont bien les inactives (2,91 enfants en moyenne) ainsi que les agricultrices (2,23 enfants en moyenne, contre 1,91 dans la population générale) (22).

(22) Le fait que les agriculteurs connaissent un écart d'âge entre conjoints relativement élevé ne saurait ici s'expliquer par le fait qu'ils passent un temps relativement long à accumuler le capital nécessaire à l'acquisition d'une exploitation - ce qui accroîtrait leur âge à la mise en couple et par suite l'écart d'âge avec leur conjointe - parce que les analyses reportées ici identifient l'effet de la profession des hommes sur l'écart d'âge à même âge à la mise en couple. 
Quoi qu'il en soit, il apparaît que l'écart d'âge tend bien à décroître avec la position sociale de l'homme du couple. En effet, mis à part les agriculteurs qui sont les hommes qui connaissent le plus fort écart d'âge -, les ouvriers connaissent un écart d'âge plus élevé que les employés et indépendants, qui eux-mêmes connaissent un écart d'âge plus élevé que les professions intermédiaires et les cadres ou professions intellectuelles supérieures. De même qu'avec le niveau de diplôme, on observe qu'avec la catégorie socioprofessionnelle des hommes l'écart d'âge entre conjoints décroît, de nos jours, à mesure que s'élève la position sociale. De ce point de vue, il est clair que le gradient social de l'écart d'âge entre conjoints qui est observé dans la France contemporaine est inverse à celui observé dans les sociétés agraires pour lesquelles des données sont disponibles : alors que des données brutes indiquent qu'il croît avec la position sociale de l'homme dans les sociétés agraires, mais que ce n'est plus le cas dans la France contemporaine, les résultats nets montrent que l'écart d'âge entre conjoints décroît avec la position sociale de l'homme. C'est la principale observation que le modèle Bergstrom-Bagnoli ne permet pas d'expliquer.

En fin de compte, ce modèle apparaît validé empiriquement dans la mesure où, dans la France contemporaine, il explique de façon satisfaisante 1) l'effet - majeur - de l'âge à la mise en couple sur l'écart d'âge entre conjoints, ainsi que 2) l'effet de l'inactivité féminine et 3) l'effet du nombre d'enfants qu'ont déjà eus les conjoints sur leur écart d'âge. Selon ce modèle, chacune de ces régularités s'explique fondamentalement par la préférence des hommes pour des femmes jeunes - physiquement plus attractives et potentiellement plus fécondes - et par la préférence des femmes pour des hommes d'âge mûr - aux revenus plus élevés et plus stables. Cela dit - et même si ce modèle permet aussi d'expliquer la direction du gradient social de l'écart d'âge entre conjoints dans les sociétés agraires -, ce modèle ne permet pas d'expliquer le retournement - et la nouvelle direction - du gradient social de l'écart d'âge entre conjoints dans la France contemporaine : seul l' « effet institution » des études semble en capacité d'expliquer pourquoi, de nos jours, les hommes plus diplômés connaissent un écart d'âge avec leur épouse plus faible que les autres. La question qui se pose, dès lors, est de savoir s'il serait scientifiquement utile de complexifier le modèle Bergstrom-Bagnoli en vue qu'il parvienne aussi à expliquer l'inversion du gradient social de l'écart d'âge due au mouvement de scolarisation. Même si la question est ouverte, il apparaît qu'étant donné le nombre de phénomènes qu'il permet d'expliquer un modèle aussi simple peut être considéré comme relativement performant.



Parvenu au terme de nos investigations, il convient d'en dresser un bilan. Dans un premier temps, nous avons établi plusieurs phénomènes empiriques : 
- il existe un écart d'âge moyen entre conjoints au profit de l'homme dans la quasi-totalité des sociétés humaines connues, quels que soient le lieu et la période ;

- au fil du temps aussi bien qu'entre sociétés, cet écart tend à décroître avec le développement socioéconomique ;

- au sein de toutes ces sociétés, l'écart d'âge moyen entre conjoints crô̂t avec l'âge de l'homme à la mise en couple et décroît avec l'âge de la femme à la mise en couple;

- au sein des sociétés agraires, cet écart croît avec la position sociale du couple.

Dans un second temps, nous avons cherché à expliquer ces phénomènes, c'est-à-dire à restituer les «bonnes raisons » que peuvent avoir les individus de former des couples comportant un écart d'âge entre conjoints au profit de l'homme. En nous appuyant sur un modèle explicatif proposé par la théorie du choix rationnel, nous avons montré que le fait qu'il existe un écart d'âge moyen entre conjoints au profit de l'homme dans la (quasi-) totalité des sociétés humaines connues semble fondamentalement dériver du fait - apparemment lui aussi universel - que les hommes préfèrent les femmes jeunes et que les femmes préfèrent les hommes d'âge mûr, et ce en raison de l'importance qu'accorderaient les hommes à l'attractivité physique ainsi qu'à la fécondité potentielle de leurs conjointes, et en raison de l'importance qu'accorderaient les femmes au niveau ainsi qu'à la stabilité des revenus ou ressources de leurs conjoints.

À cet égard, il n'est pas étonnant qu'au fil du temps aussi bien qu'entre sociétés le développement socioéconomique réduise l'écart d'âge entre conjoints. D'une part, le fait que les hommes désirent de moins en moins d'enfants réduit pour eux l'importance d'avoir pour conjointe une femme jeune ; en accord avec cette explication, nous avons observé que, dans la France contemporaine, les individus qui font le plus d'enfants font partie des catégories socioprofessionnelles connaissant les écarts d'âge les plus élevés, et nous avons observé que les hommes qui ont déjà eu des enfants connaissent dans leur nouveau couple des écarts d'âge relativement réduits. D'autre part, le fait que les femmes deviennent indépendantes économiquement réduit pour elles l'importance d'avoir pour conjoint un homme d'âge mûr ; et, de fait, dans la France contemporaine, nous avons observé que les femmes qui n'ont pas encore débuté leur carrière professionnelle tendent à choisir des hommes avec lesquels elles entretiennent un écart d'âge plus grand que celles qui l'ont déjà débutée.

De même, il n'est pas étonnant que, dans toutes les sociétés humaines analysées - et notamment, ici, la France contemporaine -, l'écart d'âge croisse avec l'âge de l'homme à la mise en couple et décroisse avec celui de la femme à la mise en couple. La préférence des hommes pour des femmes jeunes les conduit à se mettre en couple avec des femmes d'autant plus jeunes par rapport à eux qu'ils sont eux-mêmes plus âgés, et la préférence des femmes pour des hommes d'âge mûr les conduit à se mettre en couple avec des hommes d'autant moins âgés par rapport à elles qu'elles sont elles-mêmes plus âgées. 
Les recherches que nous avons menées à partir de la littérature empirique sur diverses sociétés agraires ainsi qu'à partir de modèles d'analyse de variance sur la France contemporaine indiquent, en outre, que le gradient social de l'écart d'âge entre conjoints s'est inversé. Alors que, dans les sociétés agraires, l'écart d'âge croît avec la position sociale des hommes - car ne peuvent atteindre une position sociale élevée que les hommes qui reportent leur mariage pour se constituer un capital, ce report du mariage accroissant l'écart d'âge avec leur épouse -, dans la France contemporaine, l'écart d'âge décroît à mesure que s'élève le niveau de diplôme des hommes, vraisemblablement parce que le fait de prolonger ses études accroît la probabilité de rencontrer sa conjointe sur son lieu d'études, un lieu sur lequel sont concentrées des femmes d'âges très proches. Ces observations menées sur la société française contemporaine tendent donc à indiquer que la prolongation de la scolarité des hommes, mais aussi l'entrée des femmes sur le marché du travail ainsi que la baisse de la fécondité désirée enclenchent divers mécanismes («effet institution » des études des hommes, « effet indépendance » du travail des femmes) incitant les individus à réduire l'écart d'âge au profit de l'homme au sein de leurs couples. En revanche, il n'existe pas de raison forte de penser que l'écart d'âge entre conjoints serait près de disparaître.

Jean-François MIGNOT

Observatoire sociologique du changement-Sciences Po-Cnrs 27, rue Saint-Guillaume 75337 PARIS cedex 07

Laboratoire de sociologie quantitative Centre de recherche en économie et statistique (Crest)

Insee

Timbre J 350

3, avenue Pierre Larousse 92245 Malakoff cedex 


\section{RÉFÉRENCES BIBLIOGRAPHIQUES}

Barbieri M., Hertrich V., 2005. - «Écarts d'âge entre conjoints et pratique contraceptive en Afrique subsaharienne », Population, 5/6, pp. 725-764.

Barre C., Vanderschelden M., 2004. - «L'enquête "Étude de l'histoire familiale" de 1999. Résultats détaillés », Insee résultats, 33.

Bergstrom T. C., Bagnoli M., 1993. - «Courtship as a waiting game», Journal of political economy, 101, 1, pp. 185-202.

Bergstrom T. C., Schoeni R. F., 1996. - «Income prospects and age at marriage », Journal of population economics, 9, pp. 115-130.

Biégelmann-Massari M., 1996a. - «Les dispenses civiles au mariage de 1960 à 1992. I. Le choix d'un parent pour conjoint », Population, 1, pp. 61-91.

— 1996b. - «Les dispenses civiles au mariage de 1960 à 1992. II. Le mariage posthume : mariage de raison ou mariage d'amour? ", Population, 2, pp. 369-396.

Blossfeld H.-P., 2009. - «Educational assortative marriage in comparative perspective », Annual review of sociology, 35, pp. 513-530.

Bologne J.-C., 1995. - Histoire du mariage en Occident, Paris, Hachette.

Bonneuil N., 1992. - "Démographie de la nuptialité au XIX $X^{\mathrm{e}}$ siècle» dans J. Dupâquier, D. Kessler, D. Blanchet (éds.), La société française au XIX $X^{e}$ siècle : tradition, transition, transformations, Paris, Fayard, pp. 83-119.

Bozon M., 1990a. - «Les femmes et l'écart d'âge entre conjoints : une domination consentie. I. Types d'union et attentes en matière d'écart d'âge », Population, 2, pp. 327-360.

- 1990b. - «Les femmes et l'écart d'âge entre conjoints : une domination consentie. II. Modes d'entrée dans la vie adulte et représentations du conjoint », Population, 3, pp. 565-602.

— 1991. - «Les femmes plus âgées que leur conjoint sont-elles atypiques?», Population, 1, pp. 152-159.

Bozon M., Héran F., 1988 - « La découverte du conjoint. II. Les scènes de rencontre dans l'espace social », Population, 1, pp. 121-150.

Buss D. M., 1989. - « Sex differences in human mate preferences : evolutionary hypotheses tested in 37 cultures », Behavioral and brain sciences, 12, pp. 1-49.

Cassan F., Mary-Portas F.-L., 2002. - «Précocité et instabilité familiale des hommes détenus », Insee première, 828.

Cassan F., Mazuy M., Clanché F., 2001. - «Refaire sa vie de couple est plus fréquent pour les hommes », Insee première, 797.

Casterline J. B., Williams L., McDonald P., 1986. - « The age difference between spouses : variations among developing countries », Population studies, 40, 3, pp. 353-374.

Cayemittes M., Placide M. F., Barrère B., Mariko S., Sévère B., 2001. - Enquête mortalité, morbidité et utilisation des services, Haïti 2000, Calverton (MD), Ministère de la Santé publique et de la Population, Institut haïtien de l'Enfance et ORC Macro.

Charbonneau H., 1970. - Tourouvre-au-Perche aux XVII et XVIII siècles. Étude de démographie historique, Paris, Presses Universitaires de France.

- 1980. - «Jeunes femmes et vieux maris : la fécondité des mariages précoces », Population, 6, pp. 1101-1122.

Chenu A., 2008. - «Des sentiers de la gloire aux boulevards de la célébrité. Sociologie des couvertures de Paris Match, 1949-2005 », Revue française de sociologie, 49, 1, pp. 3-52.

Choo E., Siow A., 2005. - Lifecycle marriage matching : theory and evidence, Toronto, University of Toronto [non publié].

Cohen L. R., 1987. - " Marriage, divorce, and quasi rents; or, "I gave him the best years of my life" », The journal of legal studies, 16, 2, pp. 267-303.

- 2002. - «Marriage : the long-term contract» dans A. W. Dnes, R. Rowthorn (eds.), The law and economics of marriage and divorce, Cambridge, Cambridge University Press, pp. 10-34. 
Davis A., 1998. - «Age differences in dating and marriage : reproductive strategies or social preferences ?», Current anthropology, 39, 3, pp. 374-380.

Dixon R. B., 1971. - « Explaining cross-cultural variations in age at marriage and proportion never marrying », Population studies, 25, 2, pp. 215-233.

Duchesne L., 2004. - «Quatre ans d'écart d'âge en moyenne entre les conjoints », Données sociodémographiques en bref-conditions de vie (Institut de la statistique du Québec), 8, 3, p. 1-2.

Duplessis-Le Guélinel G., 1954. - Les mariages en France, Paris, Armand Colin.

Ferrand M., 2004. - Féminin, masculin, Paris, La Découverte.

Festy P., 1971. - «Évolution de la nuptialité en Europe occidentale, depuis la guerre », Population, 2, pp. 331-379.

Gallman J. M., 1984. - « Relative ages of colonial marriages », Journal of interdisciplinary history, 14, 3, pp. 609-617.

Grange C., 1996. - Les gens du Bottin Mondain, 1903-1987. Y être, c'est en être, Paris, Fayard.

Grossbard-Shechtman S., 1993. - On the economics of marriage. A theory of marriage, labor, and divorce, Boulder (CO), Westview Press.

Guillaume P., 1972. - La population de Bordeaux au XIXe siècle. Essai d'histoire sociale, Paris, Armand Colin.

Henry L., Houdaille J., 1979. - « Célibat et âge au mariage aux XVIII et XIX ${ }^{\mathrm{e}}$ siècles en France. II. Âge au premier mariage », Population, 2, pp. 403-442.

Hertrich V., Pilon M., 1997. - «Les changements matrimoniaux en Afrique », La chronique du CEPED, 26.

Houdaille J., 1982. - « La nuptialité sous la Révolution et l’Empire », Population, 1, pp. 160-167.

— 1989. - «La noblesse française, 1600-1900», Population, 3, pp. 501-513.

Kayiba Mbelu E., 2005. - « Les causes de l'évolution de l'âge moyen au premier mariage, de l'écart d'âge entre les époux, de la dot et de la polygamie dans la ville de Kinshasa de 1967 à 2004 », $25^{\mathrm{e}}$ Congrès général de la population.

Keeley M. C., 1977. - « The economics of family formation », Economic inquiry, 15, 2, pp. 238-250.

Lakdawalla D. N., Schoeni R. F., 2003. - « Is nursing home demand affected by the decline in age difference between spouses ? », Demographic research, 8, pp. 279-304.

Le Bras H., Todd E., 1981. - L'invention de la France. Atlas anthropologique et politique, Paris, Librairie Générale Française.

Lery A., 1975. - «Données de démographie générale. Nuptialité 1931-1973 », Paris, Insee (Les collections de l'Insee, série D Démographie et emploi, 41).

Lévy C., Henry L., 1960. - « Ducs et pairs sous l'Ancien Régime. Caractéristiques démographiques d'une caste », Population, 5, pp. 807-830.

Nations Unies, 1990. - Patterns of first marriage: timing and prevalence, New York (NY), United Nations.

- 2000. - World marriage patterns 2000, New York (NY), United Nations.

- 2007. - World marriage data 2006, New York (NY), United Nations.

Oppenheimer V. K., 1988. - « A theory of marriage timing », American journal of sociology, 94, 3, pp. 563-591.

Otta E., Da Silva Queiroz R., De Sousa Campos L., Dowbor Da Silva M. W., Telles Silveira M., 1999. - «Age differences between spouses in a Brazilian marriage sample», Evolution and human behavior, 20, pp. 99-103.

Ouadah-Bedidi Z., Vallin J., 2003. - «Écarts d'âge entre conjoints en Algérie. Évolution depuis 1966 et disparités régionales », Revue européenne de démographie, 19, 3, pp. 79-302.

Prost A., 1981. - « Mariage, jeunesse et société à Orléans en 1911 », Annales ESC, 4, pp. 672-701.

Robert-Bobée I., Mazuy M., 2005. - «Calendrier de constitution des familles et âge de fin des études » dans C. Lefèvre, A. Filhon (éds.), Histoires de famille, histoires familiales, Paris, Ined (Les cahiers de l'Ined), pp. 175-200.

Saardchom N., 2001. - Marriage markets across countries, Philadelphia (PA), The Wharton School, University of Pennsylvania [non publié]. 
Sardon J.-P., 2005. - «L'évolution de la nuptialité en France » dans C. Bergouignan, C. Blayo, A. Parant, J.-P. Sardon, M. Tribalat (éds.), La population de la France. Évolutions démographiques depuis 1946, Paris, Cudep/Ined, pp. 169-216.

Singly F. (de), 1984. - « Les manœuvres de séduction : une analyse des annonces matrimoniales », Revue française de sociologie, 25, 4, pp. 523-559.

Smeenk W., 1998. - Opportunity and marriage. The impact of individual resources and marriage market structure on first marriage timing and partner choice in the Netherlands, Amsterdam, Thela Thesis.

Sohn A.-M., 1996. - Chrysalides. Femmes dans la vie privée (XIXe-XXe siècles), Paris, Publications de la Sorbonne.

Sutter J., Lévy C., 1959. - «Les dispenses civiles au mariage en France depuis 1800 », Population, 2, pp. 285-304.

Vallot F., 1971. - « Mariages et divorces à Paris : analyse des actes de mariage de quatre cohortes », Population, numéro spécial, pp. 67-100.

Van Poppel F., Liefbroer A. C., Vermunt J. K., Smeenk W., 2001. - « Love, necessity and opportunity: changing patterns of marital age homogamy in the Netherlands, 1850-1993 », Population studies, 55, 1, pp. 1-13.

Vanderschelden M., 2006. - «L'écart d'âge entre conjoints s'est réduit », Insee première, 1073.

Vernier B., 1977. - «Émigration et dérèglement du marché matrimonial », Actes de la recherche en sciences sociales, 15, pp. 31-58.

- 1985. - «Stratégies matrimoniales et choix d'objet incestueux. Dot, diplôme, liberté sexuelle, prénom », Actes de la recherche en sciences sociales, 57/58, pp. 3-27. 